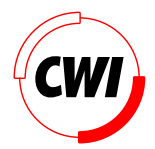

Centrum voor Wiskunde en Informatica REPORTRAPPORT

Central limit theorem for a class of random measures associated with germ-grain models

L. Heinrich and I.S. Molchanov

Department of Operations Reasearch, Statistics, and System Theory

BS-R9518 1995 
Report BS-R9518

ISSN 0924-0659

CWI

P.O. Box 94079

1090 GB Amsterdam

The Netherlands

$\mathrm{CWI}$ is the National Research Institute for Mathematics and Computer Science. CWI is part of the Stichting Mathematisch Centrum (SMC), the Dutch foundation for promotion of mathematics and computer science and their applications.

SMC is sponsored by the Netherlands Organization for Scientific Research (NWO). CWI is a member of ERCIM, the European Research Consortium for Informatics and Mathematics.

Copyright @ Stichting Mathematisch Centrum P.O. Box 94079, 1090 GB Amsterdam (NL) Kruislaan 413, 1098 SJ Amsterdam (NL) Telephone +3120 5929333 Telefax +3120 5924199 


\title{
Central Limit Theorem for a Class of Random Measures Associated with Germ-Grain Models
}

\author{
Lothar Heinrich \\ TU Bergakademie Freiberg, Institut für Stochastik \\ D-09596 Freiberg, Germany \\ heinrich@mathe.tu-freiberg.d400.de \\ Ilya S. Molchanov \\ $C W I$ \\ P.O. Box 94079, 1090 GB Amsterdam, The Netherlands \\ ilia@cwi.nl
}

\begin{abstract}
The paper introduces a family of stationary random measures in $\mathbb{R}^{d}$ generated by so-called germgrain models. The germ-grain model is defined as the union of i.i.d. compact random sets (grains) shifted by points (germs) of a point process. This model gives rise to random measures defined by the sum of contributions of non-overlapping parts of the individual grains. The corresponding moment measures are calculated and the ergodic theorem is presented. The main result of the paper is the central limit theorem for the introduced random measures, which is valid for rather general independently marked germ-grain models, including those with non-Poisson distribution of germs and non-convex grains. The technique is based on a central limit theorem for $\beta$-mixing random fields. It is shown that this construction of random measures includes those random measures obtained by the so-called positive extensions of intrinsic volumes. In the Poisson case it is possible to prove a central limit theorem under weaker assumptions by using approximations by $m$-dependent random fields. Applications to statistics of the Boolean model are also discussed. They include a standard way to derive limit theorems for estimators of the model parameters.
\end{abstract}

AMS Subject Classification (1991): 52A22, 60D05, 60F05, 60G57.

Keywords $\& 3$ Phrases: $\beta$-mixing, Boolean model, germ-grain model, intrinsic volumes, $m$-dependent random field, moment measure, probability generating functional, random measure, random set, spatial processes, surface area, weak dependence.

Notes: The second author was supported by the Netherlands Organization for Scientific Research (NWO). This paper will be submitted for publication. 


\section{INTRODUCTION}

The ergodic theorem for spatial processes [19] establishes the existence of spatial averages when a sampling window expands unboundedly in all directions. In many applications such averages can be interpreted as intensities of some stationary random measures having random closed supports in $\mathbb{R}^{d}$. For example, a point process corresponds to the measure which counts the number of elements belonging to a discrete random set, a random closed set with non-empty interior gives rise to the random volume measure. Further examples are provided by geometric measures, e.g., surface and curvature measures of random sets. The relationships between intensities of such measures and parameters of the underlying random set or point process are the basis of the method of moments in statistics of stationary random sets $[18,26]$.

A simple example states that, for any ergodic random closed set $Z$ in $\mathbb{R}^{d}$, the volume fraction inside window $W$ (the part of the window covered), converges almost surely to its expectation called the volume fraction of $Z$, while $W$ is assumed to expand to the whole space in a "regular way" [4, p. 332]. The corresponding central limit theorem was established in $[2,15]$. In fact, it is related to weak dependence properties of the corresponding indicator random field $\zeta(x)=\mathbf{1}_{Z}(x)$ with the subsequent applications of limit theorems for additive functionals of random fields.

Another example stems from the theory of point processes. If $Z$ is a point process in $\mathbb{R}^{d}$, then the corresponding counting measure gives the number of points in a bounded window. Its spatial density (for ergodic point processes) is equal to the intensity of the point process. The central limit theorem for the number of points is true under some conditions of weak dependence within $Z$. The limiting variance is related to the secondorder characteristics of $Z$.

For more general functionals (which include, e.g., the boundary length or surface area) the convergence of spatial averages was considered in [19] and [27]. However, until now a general approach to derive limit theorems for such functionals seems to be unknown. It should be noted that these limit theorems are very important for the construction of confidence intervals for estimators in statistics of stationary random sets. Sometimes it is possible to derive the asymptotic normality of the spatial averages, but the computation of the variance are usually impossible. In turn, the latter is caused by the lack of secondorder integral-geometric formulae, see [25].

The first limit theorems for geometric functionals other than volume fractions were proved in [18] and [17] for the so-called Boolean model $Z$ and functionals equal to the convexity number or the surface measure. In this paper we consider more general models of random sets and geometric functionals and prove the corresponding central limit theorem. Also, we do not need the boundedness of the grain assumed in [17] for the case of surface measures.

As known from probability theory, ergodicity and first moment assumptions are not sufficient to hold a central limit theorem. One has to impose further conditions on both the random sets and the corresponding random measures. In the present paper we are interested in a class of random measures associated with so-called independently marked germ-grain models. Nevertheless, we will show that this special class includes positive 
extensions of the intrinsic volumes, which are very important in statistics of random closed sets.

To begin with, we recall the definition of germ-grain models and introduce a class of random measures associated with them (Section 3). The germ-grain model is composed from a sequence of independent identically distributed random sets (grains) shifted by the points (germs) of a point process. Germ-grain models with compact grains are widely used in stochastic geometry and generalize the well-known Boolean model which appears if the point process of germs is Poisson. Roughly speaking, the associated random measures are defined through sums of contributions of those parts of individual grains which are exposed (not covered by all other grains). In the simplest case, this construction can be used to obtain the surface area of the germ-grain model. The associated random measures include, for instance, those Minkowski measures (or positive extensions of intrinsic volumes) defined by Matheron [16, Section 4.7].

Statistical properties of associated random measures are studied in Sections 4 and 5 . In particular, higher-order mixed moment measures are given in Section 4 and the absolute regularity (or $\beta$-mixing) is proved in Section 5 . We show that the $\beta$-mixing property of the germ process implies $\beta$-mixing of the germ-grain model and the associated random measure provided the diameter of the typical grain has sufficiently high moments. Section 6 establishes the central limit theorem for associated random measures. Section 7 deals with the Boolean model for which the central limit theorem holds under very mild (in fact, optimal) conditions.

Relationships between the introduced associated random measures and standard geometric functionals are studied in Section 8. Then the introduced associated random measures give, in particular, the so-called positive extensions of the intrinsic volumes, e.g., volume, surface area, integrals of absolute curvatures, convexity number etc. Mean value formulae for extended intrinsic volumes are very important in statistics of the Boolean model. Therefore, the generalized associated random measures give rise to new meanvalue formulae and also provide a unified approach to limit theorems for spatial averages. Such statistical applications and examples are considered in Section 9. Several possible generalizations are outlined in Section 10.

\section{List of Notations}

$$
\begin{array}{ll}
{\left[\mathbb{R}^{d}, \mathfrak{B}^{d}\right]} & : d \text {-dimensional Euclidean space with Borel } \sigma \text {-field; } \\
\mathfrak{B}_{0}^{d} & : \text { family of bounded Borel sets in } \mathbb{R}^{d} ; \\
\|x\| & : \text { Euclidean norm of } x \in \mathbb{R}^{d} ; \\
\|K\| & =\sup \{\|x\|: x \in K\}, K \in \mathfrak{B}_{0}^{d} ; \\
|B| & : d \text {-dimensional Lebesgue measure (or volume) of } B \in \mathfrak{B}_{0}^{d} ; \\
B_{r}(x) & =\left\{y \in \mathbb{R}^{d}:\|x-y\| \leq r\right\} \text { for } x \in \mathbb{R}^{d} \text { and } r>0 ; \\
\mathbf{1}_{B}(x) & =1 \text { if } x \in B \text { and }=0 \text { otherwise (indicator function of } B \text { ); } \\
F^{c} & : \text { complement of set } F \text { in the underlying space; }
\end{array}
$$


$\partial F \quad$ : boundary of $F \subset \mathbb{R}^{d}$;

$\left[\mathcal{F}, \sigma_{f}\right] \quad$ : family of closed sets in $\mathbb{R}^{d}$ with Matheron's $\sigma$-field, see [16];

$\left[\mathcal{K}, \sigma_{k}\right] \quad$ : family of compact sets in $\mathbb{R}^{d}$ with $\sigma$-field $\sigma_{k}=\left\{\mathcal{A} \cap \mathcal{K}: \mathcal{A} \in \sigma_{f}\right\} ;$

$\check{K} \quad=\{-x: x \in K\}$ for $K \in \mathcal{K}$;

$\oplus \quad$ : Minkowski addition, i.e., $F_{1} \oplus F_{2}=\left\{x+y: x \in F_{1}, y \in F_{2}\right\}$, $F_{1}, F_{2} \subset \mathbb{R}^{d}$

$\mathcal{C} \quad$ : family of convex compact sets in $\mathbb{R}^{d} ;$

$\mathcal{R} \quad$ : convex ring, i.e., family of finite unions of sets from $\mathcal{C}$;

$M \quad$ : set of locally finite measures on $\mathbb{R}^{d}$;

$\mathfrak{M} \quad: \sigma$-field generated by sets of the form $\{\mu \in M: a \leq \mu(B) \leq b\}$, $B \in \mathfrak{B}_{0}^{d}, 0 \leq a<b<\infty$;

$[\mathbb{U}, \mathfrak{U}]$

: compact metric space with the corresponding Borel $\sigma$-field;

$[\Sigma, \mathfrak{B}(\Sigma)] \quad$ : product space $\mathbb{U} \times \mathbb{R}^{d}$ with the corresponding product $\sigma$-algebra;

$M(\Sigma)$

$\mathfrak{M}(\Sigma)$

: family of locally finite measures on $\Sigma$;

: $\sigma$-field generated by sets of the form $\{\mu \in M(\Sigma): a \leq \mu(S) \leq b\}, S \in \mathfrak{B}(\Sigma), 0 \leq a<b<\infty ;$

$N$

: set of locally finite counting measures $\psi$ on $\mathbb{R}^{d}$;

$\mathfrak{N}$

: $\sigma$-field generated by sets of the form $\{\psi \in N: \psi(B)=k\}$, $B \in \mathfrak{B}_{0}^{d}, k=0,1, \ldots$

$[\Omega, \mathfrak{A}, \mathbf{P}] \quad$ : hypothetical common probability space on which all occurring random elements are defined;

$\mathbf{E}$

: expectation with respect to $\mathbf{P}$;

$\delta_{X} \quad:$ unit measure concentrated at point $X \in \mathbb{R}^{d}$;

$\Psi=\sum_{i \geq 1} \delta_{X_{i}} \quad$ : random locally finite counting measure (point process) with distribution $P$, i.e., $P(Y)=\mathbf{P}\{\Psi \in Y\}, Y \in \mathfrak{N}$;

$\lambda \quad=\mathbf{E} \Psi\left([0,1)^{d}\right)$ intensity of $\Psi$;

$G[f] \quad=\mathbf{E}\left[\prod_{i: i \geq 1} f\left(X_{i}\right)\right]$ probability generating functional of $\Psi$;

$P_{x_{1}, \ldots, x_{k}}^{!}(\cdot) \quad: k$ th-order (reduced) Palm distribution of $\Psi$ with respect to $x_{1}, \ldots, x_{k} \in \mathbb{R}^{d}$ (see [4]);

$\sum_{x_{1}, \ldots, x_{k} \in \psi}^{*} \quad$ : the sum over all $k$-tuples of pairwise distinct atoms of $\psi \in N$;

$\alpha^{(k)}\left(B_{1} \times \cdots \times B_{k}\right)=\mathbf{E} \sum_{x_{1}, \ldots, x_{k} \in \Psi}^{*} \mathbf{1}_{B_{1}}\left(x_{1}\right) \cdots \mathbf{1}_{B_{k}}\left(x_{k}\right) \quad k$ th-order factorial moment measure of $\Psi$;

$\alpha_{\text {red }}^{(2)}\left(\right.$ resp. $\left.\gamma_{\text {red }}^{(2)}\right) \quad:$ the reduced moment (respectively reduced covariance) measure of $\Psi$, i.e., $\alpha_{\text {red }}^{(2)}(B)=\int_{N} \psi(B) P_{0}^{!}(d \psi), \gamma_{\text {red }}^{(2)}(B)=\alpha_{\text {red }}^{(2)}(B)-\lambda|B|$, $B \in \mathfrak{B}_{0}^{d}$. 


\section{Germ-Grain Models and Associated Random Measures}

Many random measures in stochastic geometry are related to some stationary random sets. Stationary random closed set $Z$ is a random element in space $\left[\mathcal{F}, \sigma_{f}\right]$ such that $Z$ has the same distribution as $Z+x$ for all $x \in \mathbb{R}^{d}$. Tractable models of stationary random sets are produced by set-theoretic operations applied to some elementary components (stationary point processes and "simple" random sets). Perhaps, the most important one is the germ-grain model. This model is defined by a stationary marked point process

$$
\Psi_{m}=\sum_{i: i \geq 1} \delta_{\left[X_{i} ; Z_{i}\right]}
$$

in $\mathbb{R}^{d}$ with the mark space $\left[\mathcal{K}, \sigma_{k}\right]$, where the stationarity is understood with respect to its first component, see [23] for further details on marked point processes. The corresponding germ-grain model $Z$ is defined as the set-theoretic union

$$
Z=\bigcup_{i: i \geq 1}\left(X_{i}+Z_{i}\right)
$$

The points $X_{i}$ are called germs, while the sets $Z_{i}$ are called grains, see [8,23]. The model exists as soon as $Z$ is closed. The existence and ergodic properties of germ-grain models are studied in [11].

In this paper we will consider only independently marked point processes. Throughout we assume that the corresponding unmarked point process

$$
\Psi(\cdot)=\Psi_{m}((\cdot) \times \mathcal{K})=\sum_{i: i \geq 1} \delta_{X_{i}}
$$

is simple and has a positive and finite intensity $\lambda=\mathbf{E} \Psi\left([0,1]^{d}\right)$. Remember that independent marking is thought of as follows: $\left\{Z_{i}, i \geq 1\right\}$ is a sequence of i.i.d. copies of a random compact set (mark) $Z_{0}$ with distribution

$$
Q(\mathcal{X})=\mathbf{P}\left\{Z_{0} \in \mathcal{X}\right\}, \quad \mathcal{X} \in \sigma_{k}
$$

being independent of $\Psi$ and each atom $X_{i}$ is marked with $Z_{i}$ so that

$$
\Psi_{m}(B \times \mathcal{X})=\#\left\{i \geq 1: X_{i} \in B, Z_{i} \in \mathcal{X}\right\}, \quad B \in \mathfrak{B}_{0}^{d}, \quad \mathcal{X} \in \sigma_{k}
$$

The random compact set $Z_{0}$ is called the typical grain or the typical mark. Note that a random compact set is defined to be a random element in the space $\left[\mathcal{K}, \sigma_{k}\right],[16$, Chapter 2]. Then random set (3.1) is closed and different from $\mathbb{R}^{d}$ (so the germ-grain model exists) if

$$
\mathbf{E}\left|Z_{0} \oplus B_{r}(0)\right|<\infty \text { for } r>0
$$

(e.g., if $\left.\mathbf{E}\left\|Z_{0}\right\|^{d}<\infty\right)$, see [11].

In case the point process of germs $\Psi$ is Poisson, $Z$ is said to be a Boolean model. This is the best studied and most applied model of a stationary random closed set, see [23].

Germ-grain models give rise to several random measures, see [16]. In the simplest case it is possible to put $\eta(W)=|Z \cap W|$. Then $\eta$ is a random measure on $\mathfrak{B}_{0}^{d}$. Another 
random measure can be defined by taking the surface area of $Z$ inside $W$. Further random measures are the so-called Minkowski random measures introduced in [16]. It is worthwhile to note that many interesting geometric measures associated with $Z$ can be decomposed into the sum with respect to all individual grains. In this sum each grain contributes to the resulting measure with some (possibly random) weight. For further information on random measures the reader is referred to [14], [4, Chapter 6] and [23].

Below we present a unified approach to random measures associated with germ-grain models and marked point processes. First, define

$$
H_{\bullet}: \mathcal{K} \mapsto M(\Sigma)
$$

to be a $\left(\sigma_{k}, \mathfrak{M}(\Sigma)\right)$-measurable mapping. For each $K \in \mathcal{K}, H_{K}(\cdot)$ is a finite measure on $\mathfrak{B}(\Sigma)$. For the sake of convenience we mostly write $H_{K}(\Gamma, W)$ instead of $H_{K}(\Gamma \times W)$ for $\Gamma \in \mathfrak{U}$ and $W \in \mathfrak{B}_{0}^{d}$. We assume that this measure-valued map satisfies the following conditions

$$
H_{K}(\Gamma, W)=H_{K}(\Gamma, W \cap K)
$$

and

$$
H_{K+x}(\Gamma, W+x)=H_{K}(\Gamma, W)
$$

for all $K \in \mathcal{K}, \Gamma \in \mathfrak{U}, W \in \mathfrak{B}_{0}^{d}$, and $x \in \mathbb{R}^{d}$.

Moreover, we assume that

$$
\mathbf{E} H_{Z_{0}}\left(\mathbb{U}, \mathbb{R}^{d}\right)<\infty,
$$

so that

$$
\bar{H}(\Gamma, W)=\mathbf{E} H_{Z_{0}}(\Gamma, W)
$$

is a finite deterministic measure on $\mathfrak{B}(\Sigma)$.

Remark 1. It is also possible to consider signed measures $H$. Then the finiteness condition must be replaced by the finiteness of the expected total variation of $H_{Z_{0}}$.

We are now in a position to define a locally finite random measure $\eta(\Gamma, W)$ on $\mathfrak{B}(\Sigma)$ by

$$
\eta(\Gamma, W)=\sum_{i: i \geq 1} H_{X_{i}+Z_{i}}\left(\Gamma, W \backslash \Xi_{i}\right), \quad \Gamma \in \mathfrak{U}, W \in \mathfrak{B}_{0}^{d}
$$

where

$$
\Xi_{i}=\bigcup_{j: i \neq i}\left(X_{j}+Z_{j}\right), \quad i \geq 1
$$

Note that $H_{K}(\cdot)$ can be identically zero for some $K$. This means that the corresponding terms in (3.7) do not contribute to the sum.

The local finiteness of $\eta$ results from conditions ensuring the closedness of the germgrain model, for example, (3.2) is sufficient. Obviously, $\eta(\Gamma, W+x)$ equals $\eta(\Gamma, W)$ in distribution for all $\Gamma \in \mathfrak{U}, W \in \mathfrak{B}_{0}^{d}$, and $x \in \mathbb{R}^{d}$, i.e., $\eta(\Gamma, \cdot)$ is a stationary random measure on $\mathfrak{B}_{0}^{d}$.

To avoid confusion, we put together our basic assumptions. 


\section{Basic assumptions:}

(i) $\Psi_{m}$ is a simple, stationary, independently marked point process with a finite positive intensity $\lambda>0$.

(ii) The measurable mapping $K \mapsto H_{K}(\cdot)$ satisfies (3.3) and (3.4).

(iii) The typical grain $Z_{0}$ satisfies $(3.2)$ and (3.5).

Now consider an important example of measures $H_{K}(\cdot)$.

EXAmple 3.1. Consider family of measures $H_{K}(\cdot), K \in \mathcal{K}$, which admit integral representation

$$
H_{K}(\Gamma, W)=\int_{\Gamma} \mathbf{1}_{W}(\ell(u, K)) \Upsilon_{K}(d u), \quad K \in \mathcal{K},
$$

where $\Upsilon_{\bullet}: \mathcal{K} \mapsto M(\mathbb{U})$ is a $\left(\sigma_{k}, \mathfrak{M}(\mathbb{U})\right)$-measurable mapping, where $M(\mathbb{U})$ is the set of finite measures on $[\mathbb{U}, \mathfrak{U}]$, and $\mathfrak{M}(\mathbb{U})$ is the $\sigma$-field generated by sets of the form $\{\mu \in$ $M(\mathbb{U}): a \leq \mu(U) \leq b\}, U \in \mathfrak{U}, 0 \leq a<b<\infty$. Assume that $\Upsilon_{K}(\cdot)$ is invariant under shifts of $K$, i.e., $\Upsilon_{K}(\cdot)=\Upsilon_{K+x}(\cdot)$, and the mapping $\ell: \mathbb{U} \times \mathcal{K} \mapsto \mathbb{R}^{d}$ is $\left(\mathfrak{U} \otimes \sigma_{k}, \mathfrak{B}^{d}\right)$ measurable and satisfies the conditions

$$
\ell(u, K) \in K \quad \text { and } \quad \ell(u, K+x)=\ell(u, K)+x
$$

for all $K \in \mathcal{K}, u \in \mathbb{U}$ and $x \in \mathbb{R}^{d}$. If $H_{K}(\cdot)$ admits an integral representation, then

$$
H_{K}\left(\Gamma, \mathbb{R}^{d}\right)=\Upsilon_{K}(\Gamma), \quad K \in \mathcal{K},
$$

and (3.7) can be written as

$$
\eta(\Gamma, W)=\sum_{i: i \geq 1} \int_{\Gamma} \mathbf{1}_{W}\left(X_{i}+\ell\left(u, Z_{i}\right)\right) \prod_{j: j \neq i}\left(1-\mathbf{1}_{X_{j}+Z_{j}}\left(X_{i}+\ell\left(u, Z_{i}\right)\right)\right) \Upsilon_{Z_{i}}(d u)
$$

In this case the following useful lemma holds.

Lemma 3.2. Suppose that $H_{K}(\cdot)$ admits integral representation (3.9). Then, for any measurable function $f: \mathbb{R}^{d} \mapsto[0, \infty)$,

$$
\mathbf{E} \int_{W} f(y) H_{Z_{0}}(\Gamma, d y)=\int_{W} f(y) \bar{H}(\Gamma, d y)=\int_{\mathcal{K}} \int_{\mathbb{R}^{d}} \mathbf{1}_{W}(\ell(u, K)) f(\ell(u, K)) \Upsilon_{K}(d u) Q(d K) .
$$




\section{Moment Measures of $\eta$}

Below we will find the moment measures of the random measure $\eta$ given by (3.7). The following lemma gives the first moment measure.

Lemma 4.1. Under the basic assumptions,

$$
\mathbf{E} \eta(\Gamma, W)=\lambda(\Gamma)|W|
$$

where

$$
\lambda(\Gamma)=\lambda \int_{\mathbb{R}^{d}} G_{0}^{!}\left[\mathbf{P}\left\{(\cdot) \notin \check{Z}_{0}+y\right\}\right] \bar{H}(\Gamma, d y) .
$$

In particular, if $\Psi$ is a stationary Poisson process with intensity $\lambda$, then

$$
\lambda(\Gamma)=\lambda \exp \left\{-\lambda \mathbf{E}\left|Z_{0}\right|\right\} \bar{H}\left(\Gamma, \mathbb{R}^{d}\right)=\lambda(1-p) \bar{H}\left(\Gamma, \mathbb{R}^{d}\right)
$$

where $p=\mathbf{P}\{0 \in Z\}$ is the volume fraction of the corresponding Boolean model $Z$.

Proof. Note that

$$
H_{X_{i}+Z_{i}}(\Gamma, W)=H_{Z_{i}}\left(\Gamma, W-X_{i}\right)=\int_{\mathbb{R}^{d}} \mathbf{1}_{W}\left(X_{i}+y\right) H_{Z_{i}}(\Gamma, d y) .
$$

Using the refined Campbell theorem [4, p. 116] and the independent marking we get

$$
\begin{aligned}
\mathbf{E} \eta & (\Gamma, W) \\
& =\mathbf{E} \sum_{i: i \geq 1} \int_{\mathbb{R}^{d}} \mathbf{1}_{W}\left(X_{i}+y\right) \prod_{j: j \neq i}\left(1-\mathbf{1}_{X_{j}+Z_{j}}\left(X_{i}+y\right)\right) H_{Z_{i}}(\Gamma, d y) \\
& =\int_{N} \sum_{i: x_{i} \in \psi} \int_{\mathcal{K}} \int_{\mathbb{R}^{d}} \mathbf{1}_{W}\left(x_{i}+y\right) \prod_{j: x_{j} \in \psi-\delta_{x_{i}}} \mathbf{E}\left(1-\mathbf{1}_{x_{j}+Z_{0}}\left(x_{i}+y\right)\right) H_{K}(\Gamma, d y) Q(d K) P(d \psi) \\
& =\lambda \iint_{\mathbb{R}^{d}} \int_{\mathbb{R}^{d}} \mathbf{1}_{W}(x+y) \int_{N} \prod_{j: x_{j} \in \psi} \mathbf{E}\left(1-\mathbf{1}_{\check{Z}_{0}}\left(x_{j}-x-y\right)\right) P_{x}^{!}(d \psi) H_{K}(\Gamma, d y) Q(d K) d x \\
& =\lambda \int_{\mathbb{R}^{d}} \int_{\mathcal{K}_{\mathbb{R}^{d}}} \int_{\mathbf{1}_{W}}(x+y) \int_{N} \prod_{j: x_{j} \in \psi} \mathbf{E}\left(1-\mathbf{1}_{\check{Z}_{0}}\left(x_{j}-y\right)\right) P_{0}^{!}(d \psi) H_{K}(\Gamma, d y) Q(d K) d x .
\end{aligned}
$$

Now, by definition of the probability generating functional $G_{0}^{!}$with respect to the Palm distribution $P_{0}^{!}$,

$$
\begin{aligned}
\int_{N} \prod_{j: x_{j} \in \psi} \mathbf{E}\left(1-\mathbf{1}_{\check{Z}_{0}}\left(x_{i}-y\right)\right) P_{0}^{!}(d \psi) & =\int_{N} \prod_{j: x_{j} \in \psi} \mathbf{P}\left\{x_{j} \notin \check{Z}_{0}+y\right\} P_{0}^{!}(d \psi) \\
& =G_{0}^{!}[f((\cdot)-y)]
\end{aligned}
$$

where

$$
f(x)=\mathbf{P}\left\{x \notin \check{Z}_{0}\right\}, \quad x \in \mathbb{R}^{d}
$$


Thus,

$$
\begin{aligned}
\mathbf{E} \eta(\Gamma, W) & =\lambda|W| \int_{\mathcal{K}} \int_{\mathbb{R}^{d}} G_{0}^{!}[f((\cdot)-y)] H_{K}(\Gamma, d y) Q(d K) \\
& =\lambda|W| \int_{\mathbb{R}^{d}} G_{0}^{!}[f((\cdot)-y)] \bar{H}(\Gamma, d y) .
\end{aligned}
$$

This proves (4.1) and (4.2). If $\Psi$ is a Poisson process, then, by Slivnyak's theorem [4, p. 459],

$$
G_{0}^{!}[f((\cdot)-y)]=G[f((\cdot)-y)]=\exp \left\{-\lambda \int_{\mathbb{R}^{d}}(1-f(x-y)) d x\right\} .
$$

By standard manipulations and Fubini's theorem,

$$
\int_{\mathbb{R}^{d}}(1-f(x-y)) d x=\int_{\mathbb{R}^{d}} \mathbf{P}\left\{x \in \check{Z}_{0}\right\} d x=\mathbf{E}\left|Z_{0}\right|
$$

proving (4.3) and completing the proof of Lemma 4.1.

If $H$ admits integral representation (3.9), then, by Lemma 3.2,

$$
\left.\lambda(\Gamma)=\lambda \int_{\mathcal{K}} \int_{\Gamma} G_{0}^{!}\left[\mathbf{P}\left\{(\cdot) \notin \check{Z}_{0}+\ell(u, K)\right)\right\}\right] \Upsilon_{K}(d u) Q(d K) .
$$

We proceed with the following general result, which gives higher-order mixed moment measures of $\eta$.

Lemma 4.2. In addition to the above basic assumptions let the $k$ th-order factorial moment measure $\alpha^{(k)}$ of $\Psi$ exist, $\mathbf{E} H_{Z_{0}}^{k}\left(\mathbb{U}, \mathbb{R}^{d}\right)<\infty$, and

$$
\int_{\left(\mathbb{R}^{d}\right)^{\nu}} \prod_{j=1}^{\nu} \mathbf{E}\left[H_{Z_{0}}^{k_{j}}\left(\mathbb{U}, B_{r}\left(-x_{j}\right)\right)\right] \alpha^{(\nu)}\left(d\left(x_{1}, \ldots, x_{k}\right)\right)<\infty
$$

for all $1 \leq \nu \leq k$ and positive numbers $k_{1}, \ldots, k_{\nu}$ satisfying $k_{1}+\cdots+k_{\nu}=k$.

Then, for every $\Gamma_{1}, \ldots, \Gamma_{k} \in \mathfrak{U}$ and $W_{1}, \ldots, W_{k} \in \mathfrak{B}_{0}^{d}$,

$$
\begin{aligned}
& \mathbf{E}\left[\eta\left(\Gamma_{1}, W_{1}\right) \cdots \eta\left(\Gamma_{k}, W_{k}\right)\right] \\
& =\sum_{\nu=1}^{k} \sum_{I_{1} \cup \ldots \cup I_{\nu}=\{1, \ldots, k\}} \int_{\left(\mathbb{R}^{d}\right)^{\nu}} \int_{(\mathcal{K})^{\nu}} \int_{\left(\mathbb{R}^{d}\right)^{k}} \prod_{j=1}^{\nu} \prod_{i_{j} \in I_{j}} \mathbf{1}_{W_{i_{j}}}\left(x_{j}+y_{i_{j}}\right) \\
& \quad \times \prod_{\substack{i, j=1 \\
i \neq j}}^{\nu} \prod_{i_{j} \in I_{j}}\left(1-\mathbf{1}_{\check{K}_{i}+y_{i_{j}}}\left(x_{i}-x_{j}\right)\right) G_{x_{1}, \ldots, x_{\nu}}^{!}\left[\mathbf{P}\left\{(\cdot) \notin \bigcup_{j=1}^{\nu} \bigcup_{i_{j} \in I_{j}}\left(\check{Z}_{0}+x_{j}+y_{i_{j}}\right)\right\}\right] \\
& \quad \times \prod_{j=1}^{\nu} \prod_{i_{j} \in I_{j}} H_{K_{j}}\left(\Gamma_{i_{j}}, d y_{i_{j}}\right) \prod_{j=1}^{\nu} Q\left(d K_{j}\right) \alpha^{(\nu)}\left(d\left(x_{1}, \ldots, x_{\nu}\right)\right) .
\end{aligned}
$$

Here the sum $\sum_{I_{1} \cup \ldots \cup I_{\nu}=\{1, \ldots, k\}}$ is taken over all non-empty partitions of the set $\{1, \ldots, k\}$ into $\nu \in\{1, \ldots, k\}$ subsets $I_{1}, \ldots, I_{\nu}$ and $G_{x_{1}, \ldots, x_{\nu}}^{!}$denotes the probability generating functional with respect to the Palm distribution $P_{x_{1}, \ldots, x_{\nu}}^{!}$. 
Remark 2. If $\Psi$ is a stationary Poisson process, then $\alpha^{(\nu)}\left(d\left(x_{1}, \ldots, x_{\nu}\right)\right)=\lambda^{\nu} d\left(x_{1}, \ldots, x_{\nu}\right)$ and

$$
G_{x_{1}, \ldots, x_{\nu}}^{!}\left[\mathbf{P}\left\{(\cdot) \notin \bigcup_{j=1}^{\nu} \bigcup_{i_{j} \in I_{j}}\left(\check{Z}_{0}+x_{j}+y_{i_{j}}\right)\right\}\right]=\exp \left\{-\lambda \mathbf{E}\left|\bigcup_{j=1}^{\nu} \bigcup_{i_{j} \in I_{j}}\left(\check{Z}_{0}+x_{j}+y_{i_{j}}\right)\right|\right\} .
$$

Condition (4.5) follows from $\mathbf{E} H_{Z_{0}}^{k}\left(\mathbb{U}, \mathbb{R}^{d}\right)<\infty$.

ProOF. For any $k$-tuple of functions $f_{j}: \mathbb{R}^{d} \mapsto \mathbb{R}^{1}, j=1, \ldots, k$, with bounded supports the identity

$$
\sum_{x_{1}, \ldots, x_{k} \in \psi} f_{1}\left(x_{1}\right) \cdots f_{k}\left(x_{k}\right)=\sum_{\nu=1}^{k} \sum_{I_{1} \cup \ldots \cup I_{\nu}=\{1, \ldots, k\}} \sum_{y_{1}, \ldots, y_{\nu} \in \psi}^{*} \prod_{j=1}^{\nu} \prod_{i_{j} \in I_{j}} f_{i_{j}}\left(y_{j}\right)
$$

holds, where $\sum^{*}{ }_{y_{1}, \ldots, y_{\nu} \in \psi}$ is taken over all $\nu$-tuples of pairwise distinct atoms of $\psi \in N$. This identity enables us to write

$$
\begin{aligned}
\mathbf{E}\left[\eta\left(\Gamma_{1}, W_{1}\right) \cdots\right. & \left.\cdots\left(\Gamma_{k}, W_{k}\right)\right] \\
= & \sum_{\nu=1}^{k} \sum_{I_{1} \cup \ldots \cup I_{\nu}=\{1, \ldots, k\}} \mathbf{E}\left[\int_{\left.\mathbb{R}^{d}\right)^{k}} \sum_{q_{1}, \ldots, q_{\nu} \geq 1}^{*} \prod_{j=1}^{\nu} \prod_{i_{j} \in I_{j}} \mathbf{1}_{W_{i_{j}}}\left(X_{q_{j}}+y_{i_{j}}\right)\right. \\
& \left.\times \prod_{j=1}^{\nu} \prod_{\substack{p_{j} \geq 1 \\
p_{j} \neq q_{j}}} \prod_{i_{j} \in I_{j}}\left(1-\mathbf{1}_{Z_{p_{j}}-y_{i_{j}}}\left(X_{q_{j}}-X_{p_{j}}\right)\right) \prod_{j=1}^{\nu} \prod_{i_{j} \in I_{j}} H_{Z_{q_{j}}}\left(\Gamma, d y_{i_{j}}\right)\right] .
\end{aligned}
$$

By simple manipulations it is seen that, for fixed $q_{1}, \ldots, q_{\nu}$ and $I_{1}, \ldots, I_{\nu}$, the product

$$
\prod_{j=1}^{\nu} \prod_{\substack{p_{j} \geq 1 \\ p_{j} \neq q_{j}}} \prod_{i_{j} \in I_{j}}\left(1-\mathbf{1}_{Z_{p_{j}}-y_{i_{j}}}\left(X_{q_{j}}-X_{p_{j}}\right)\right)=\prod_{j=1}^{\nu} \prod_{\substack{p_{j} \geq 1 \\ p_{j} \neq q_{j}}} \prod_{i_{j} \in I_{j}}\left(1-\mathbf{1}_{\check{Z}_{p_{j}}+y_{i_{j}}}\left(X_{p_{j}}-X_{q_{j}}\right)\right)
$$

equals

$$
\prod_{j=1}^{\nu} \prod_{\substack{j=1 \\ j \neq i}}^{\nu} \prod_{i_{j} \in I_{j}}\left(1-\mathbf{1}_{\check{Z}_{q_{i}}+y_{i_{j}}}\left(X_{q_{i}}-X_{q_{j}}\right)\right) \prod_{\substack{p \geq 1 \\ p \neq q_{1}, \ldots, q_{\nu}}} \prod_{j=1}^{\nu} \prod_{i_{j} \in I_{j}}\left(1-\mathbf{1}_{\check{Z}_{p}+y_{i_{j}}}\left(X_{p}-X_{q_{j}}\right)\right) .
$$

Exploiting the independence of marks associated with distinct atoms and the independence between the marks and the point process $\Psi$ combined with a multiple use of Fubini's theorem we find that

$$
\begin{aligned}
\mathbf{E} & {\left[\sum_{q_{1}, \ldots, q_{\nu} \geq 1}^{*} \int_{\left(\mathbb{R}^{d}\right)^{k}} \prod_{j=1}^{\nu} \prod_{i_{j} \in I_{j}} \mathbf{1}_{W_{i_{j}}}\left(X_{q_{j}}+y_{i_{j}}\right) \prod_{\substack{i, j=1 \\
i \neq j}}^{\nu} \prod_{i_{j} \in I_{j}}\left(1-\mathbf{1}_{\check{Z}_{q_{i}}+y_{i_{j}}}\left(X_{q_{i}}-X_{q_{j}}\right)\right)\right.} \\
& \left.\times \prod_{\substack{p \geq 1 \\
p \neq q_{1}, \ldots, q_{\nu}}} \prod_{j=1}^{\nu} \prod_{i_{j} \in I_{j}}\left(1-\mathbf{1}_{\check{Z}_{p}+y_{i_{j}}}\left(X_{p}-X_{q_{j}}\right)\right) \prod_{j=1}^{\nu} \prod_{i_{j} \in I_{j}} H_{Z_{q_{j}}}\left(\Gamma, d y_{i_{j}}\right)\right]=
\end{aligned}
$$




$$
\begin{aligned}
= & \int_{\left(\mathbb{R}^{d}\right)^{\nu}} \mathbf{E} \int_{\left(\mathbb{R}^{d}\right)^{k}} \prod_{j=1}^{\nu} \prod_{i_{j} \in I_{j}} \mathbf{1}_{W_{i_{j}}}\left(x_{j}+y_{i_{j}}\right) \prod_{\substack{i, j=1 \\
i \neq j}}^{\nu} \prod_{i_{j} \in I_{j}}\left(1-\mathbf{1}_{\check{Z}_{i}+y_{i_{j}}}\left(x_{i}-x_{j}\right)\right) \\
& \times \int_{N} \prod_{y \in \psi} \mathbf{E}_{Z_{0}}\left[\prod_{j=1}^{\nu} \prod_{i_{j} \in I_{j}}\left(1-\mathbf{1}_{\check{Z}_{0}+y_{i_{j}}}\left(y-x_{j}\right)\right)\right] \mathbf{P}_{x_{1}, \ldots, x_{\nu}}^{!}(d \psi) \mathbf{E}\left[\prod_{j=1}^{\nu} \prod_{i_{j} \in I_{j}} H_{Z_{j}}\left(\Gamma, d y_{i_{j}}\right)\right] \\
& \times \alpha^{(\nu)}\left(d\left(x_{1}, \ldots, x_{\nu}\right)\right) .
\end{aligned}
$$

Here we have also used the relationship

$$
\begin{aligned}
\int_{N} & \sum_{x_{1}, \ldots, x_{\nu} \in \psi}^{*} f\left(x_{1}, \ldots, x_{\nu}, \psi-\delta_{x_{1}}-\ldots-\delta_{x_{\nu}}\right) P(d \psi) \\
\quad & =\int_{\left(\mathbb{R}^{d}\right)^{\nu}} \int_{N} f\left(x_{1}, \ldots, x_{\nu}, \psi\right) \mathbf{P}_{x_{1}, \ldots, x_{\nu}}^{!}(d \psi) \alpha^{(\nu)}\left(d\left(x_{1}, \ldots, x_{\nu}\right)\right)
\end{aligned}
$$

which is valid for any $\left(\mathfrak{B}^{d}\right)^{\nu} \otimes \mathfrak{N}$-measurable function $f:\left(\mathbb{R}^{d}\right)^{\nu} \times N \mapsto \mathbb{R}^{1}$, see $[4,23]$.

Finally, since

$$
\begin{aligned}
\mathbf{E}_{Z_{0}}\left[\prod_{j=1}^{\nu} \prod_{i_{j} \in I_{j}}\left(1-\mathbf{1}_{\check{Z}_{0}+y_{i_{j}}}\left(y-x_{j}\right)\right)\right] & =\mathbf{E}_{Z_{0}}\left[\prod_{j=1}^{\nu} \prod_{i_{j} \in I_{j}} \mathbf{1}_{\check{Z}_{0}^{c}+y_{i_{j}}+x_{j}}(y)\right] \\
& =\mathbf{P}_{Z_{0}}\left\{y \notin \bigcup_{j=1}^{\nu} \bigcup_{i_{j} \in I_{j}}\left(\check{Z}_{0}+y_{i_{j}}+x_{j}\right)\right\},
\end{aligned}
$$

the definition of the probability generating functional

$$
G_{x_{1}, \ldots, x_{\nu}}^{!}\left[\mathbf{P}_{Z_{0}}\left\{(\cdot) \in \bigcup_{j=1}^{\nu} \bigcup_{i_{j} \notin I_{j}}\left(\check{Z}_{0}+y_{i_{j}}+x_{j}\right)\right\}\right]
$$

yields (4.6). The liberal use of Fubini's theorem is justified because the right-hand side of (4.6) is bounded by

$$
\sum_{\nu=1}^{k} \sum_{\substack{k_{1}+\ldots+k_{\nu}=k \\ k_{i} \geq 1}} \frac{k !}{k_{1} ! \cdots k_{\nu} !} \int_{\left(\mathbb{R}^{d}\right)^{\nu}} \prod_{j=1}^{\nu} \mathbf{E}\left[H_{Z_{0}}^{k_{j}}\left(\mathbb{U}, B_{r}\left(-x_{j}\right)\right)\right] \alpha^{(\nu)}\left(d\left(x_{1}, \ldots, x_{\nu}\right)\right),
$$

where $r>0$ is chosen such that $W_{1} \cup \cdots \cup W_{k} \subseteq B_{r}(0)$. Since, by our assumptions, the latter sum is finite, Lemma 4.2 is proved.

If the grain $Z_{0}$ is Hausdorff rectifiable $\left(\mathcal{H}^{m}\right.$-rectifiable) [28] with $m<d$, then $Z$ represents a particular class of the random processes of Hausdorff rectifiable closed sets in $\mathbb{R}^{d}$. This concept includes the well-known fibre and surface processes studied in [23]. Lemma 4.2 can be used to express the corresponding moment measures found in [28].

Now we will specify Lemma 4.2 to compute second-order moment measures of $\eta$. They depend on the second-order moment measure

$$
\bar{H}\left(\Gamma_{1}, W_{1} ; \Gamma_{2}, W_{2}\right)=\mathbf{E}\left[H_{Z_{0}}\left(\Gamma_{1}, W_{1}\right) H_{Z_{0}}\left(\Gamma_{2}, W_{2}\right)\right], \quad W_{1}, W_{2} \in \mathfrak{B}_{0}^{d},
$$


of $H_{Z_{0}}(\cdot)$, and the measure

$$
\begin{aligned}
& \Phi\left(x ; \Gamma_{1}, W_{1} ; \Gamma_{2}, W_{2}\right) \\
& \quad=\mathbf{E}\left[\int_{W_{1}} \int_{W_{2}}\left(1-\mathbf{1}_{Z_{1}}\left(y_{2}+x\right)\right)\left(1-\mathbf{1}_{Z_{2}}\left(y_{1}-x\right)\right) H_{Z_{1}}\left(\Gamma_{1}, d y_{1}\right) H_{Z_{2}}\left(\Gamma_{2}, d y_{2}\right)\right] \\
& \quad=\mathbf{E}\left[H_{Z_{1}}\left(\Gamma_{1},\left(Z_{2}^{c}+x\right) \cap W_{1}\right) H_{Z_{2}}\left(\Gamma_{2},\left(Z_{1}^{c}-x\right) \cap W_{2}\right)\right]
\end{aligned}
$$

defined for two independent grains $Z_{1}$ and $Z_{2}$ having the same distribution.

Corollary 4.3. If $\Psi$ is a second-order point process, $\mathbf{E} H_{Z_{0}}^{2}\left(\mathbb{U}, \mathbb{R}^{d}\right)<\infty$ and

$$
\int_{\left(\mathbb{R}^{d}\right)^{2}} \bar{H}\left(\mathbb{U}, W-x_{1}\right) \bar{H}\left(\mathbb{U}, W-x_{2}\right) \alpha^{(2)}\left(d\left(x_{1}, x_{2}\right)\right)<\infty
$$

then

$$
\begin{gathered}
\mathbf{E}\left[\eta\left(\Gamma_{1}, W\right) \eta\left(\Gamma_{2}, W\right)\right] \\
=\lambda \int_{\left(\mathbb{R}^{d}\right)^{2}} \gamma_{W}\left(y_{2}-y_{1}\right) G_{0}^{!}\left[\mathbf{P}\left\{(\cdot) \notin\left(\check{Z}_{0}+y_{1}\right) \cup\left(\check{Z}_{0}+y_{2}\right)\right\}\right] \bar{H}\left(\Gamma_{1}, d y_{1} ; \Gamma_{2}, d y_{2}\right) \\
+\lambda \int_{\mathbb{R}^{d}\left(\mathbb{R}^{d}\right)^{2}} \gamma_{W}\left(x+y_{2}-y_{1}\right) G_{0, x}^{!}\left[\mathbf{P}\left\{(\cdot) \notin\left(\check{Z}_{0}+y_{1}\right) \cup\left(\check{Z}_{0}+x+y_{2}\right)\right\}\right] \\
\times \Phi\left(x ; \Gamma_{1}, d y_{1} ; \Gamma_{2}, d y_{2}\right) \alpha_{\text {red }}^{(2)}(d x)
\end{gathered}
$$

where $\gamma_{W}(x)=|W \cap(W+x)|$ is the set-covariance function of $W$.

Corollary 4.4. If $H$ admits integral representation (3.9), and the conditions of Corollary 4.3 are valid, then

$$
\begin{aligned}
& \mathbf{E}\left[\eta\left(\Gamma_{1}, W\right) \eta\left(\Gamma_{2}, W\right)\right] \\
& =\lambda \iint_{\mathcal{K}} \int_{\Gamma_{1}} \gamma_{W}\left(\ell\left(u_{2}, K\right)-\ell\left(u_{1}, K\right)\right) \\
& \quad \times G_{0}^{!}\left[\mathbf{P}\left\{(\cdot) \notin\left(\check{Z}_{0}+\ell\left(u_{1}, K\right)\right) \cup\left(\check{Z}_{0}+\ell\left(u_{2}, K\right)\right)\right\}\right] \Upsilon_{K}\left(d u_{1}\right) \Upsilon_{K}\left(d u_{2}\right) Q(d K) \\
& +\lambda \int_{\mathbb{R}^{d}} \int_{\mathcal{K}} \int_{\mathcal{K}} \int_{\Gamma_{1}} \int_{\Gamma_{2}} \gamma_{W}\left(x+\ell\left(u_{2}, K_{2}\right)-\ell\left(u_{1}, K_{1}\right)\right)\left(1-\mathbf{1}_{\check{K}_{1}+\ell\left(u_{2}, K_{2}\right)}(-x)\right) \\
& \quad \times\left(1-\mathbf{1}_{\check{K}_{2}+\ell\left(u_{1}, K_{1}\right)}(x)\right) G_{0, x}^{!}\left[\mathbf{P}\left\{(\cdot) \notin\left(\check{Z}_{0}+\ell\left(u_{1}, K_{1}\right)\right) \cup\left(\check{Z}_{0}+x+\ell\left(u_{2}, K_{2}\right)\right)\right\}\right] \\
& \quad \times \alpha_{\text {red }}^{(2)}(d x) \Upsilon_{K_{1}}\left(d u_{1}\right) \Upsilon_{K_{2}}\left(d u_{2}\right) Q\left(d K_{1}\right) Q\left(d K_{2}\right) .
\end{aligned}
$$


5. Absolute Regularity of the Random Measure $\eta(\Gamma, \cdot)$

Let us recall the definition of the absolute regularity coefficient (or $\beta$-mixing or weak Bernoulli coefficient) $\beta(\mathfrak{X}, \mathfrak{Y})$ between any two sub- $\sigma$-fields $\mathfrak{X}, \mathfrak{Y} \subset \mathfrak{A}[24]$

$$
\begin{aligned}
\beta(\mathfrak{X}, \mathfrak{Y}) & =\sup _{C \in \mathfrak{X} \otimes \mathfrak{Y}}\left|\mathbf{P}_{\mathfrak{X} \otimes \mathfrak{Y}}(C)-\left(\mathbf{P}_{\mathfrak{X}} \times \mathbf{P}_{\mathfrak{Y}}\right)(C)\right| \\
& =\frac{1}{2} \sup \sum_{k} \sum_{l}\left|\mathbf{P}\left(A_{k} \cap B_{l}\right)-\mathbf{P}\left(A_{k}\right) \mathbf{P}\left(B_{l}\right)\right| .
\end{aligned}
$$

Here $\mathbf{P}_{\mathfrak{X} \otimes \mathfrak{Y}}$ (respectively $\mathbf{P}_{\mathfrak{X}}$ and $\mathbf{P}_{\mathfrak{Y}}$ ) denotes the restriction to the product $\sigma$-field $\mathfrak{X} \otimes \mathfrak{Y}$ (respectively $\mathfrak{X}$ and $\mathfrak{Y}$ ) of the measure on $\Omega \times \Omega$ induced by $\mathbf{P}$ and the diagonal mapping $\omega \mapsto(\omega, \omega)$. The supremum in the second formula is taken over all pairs of finite partitions $\left\{A_{k}\right\}$ and $\left\{B_{l}\right\}$ of $\Omega$ such that $A_{k} \in \mathfrak{X}$ and $B_{l} \in \mathfrak{Y}$. Standard measure-theoretic arguments ensure that the supremum in (5.1) does not change its value when the $A_{k}$ 's and $B_{l}$ 's belong to semi-algebras $\tilde{\mathfrak{X}}$ and $\tilde{\mathfrak{Y}}$ generating $\mathfrak{X}$ and $\mathfrak{Y}$ respectively.

To be general enough, we consider the random measure

$$
\eta(f, \cdot)=\sum_{i: i \geq 1} \int_{\mathbb{U}} f(u) H_{Z_{i}}\left(d u,(\cdot) \backslash \Xi_{i}\right)
$$

for any measurable, bounded function $f: \mathbb{U} \mapsto[0, \infty)$. For any $B \in \mathfrak{B}^{d}$ define sub- $\sigma$ fields $\mathfrak{A}_{\eta(f)}(B)$ (respectively $\mathfrak{A}_{\Psi}(B)$ ) containing all events of the form $\left\{\eta\left(\Gamma, B^{\prime}\right) \in[a, b)\right\}$ (respectively $\left\{\Psi\left(B^{\prime}\right)=k\right\}$ ) for $B^{\prime} \in \mathfrak{B}_{0}^{d}, B^{\prime} \subseteq B, \Gamma \in \mathfrak{U}, 0 \leq a<b<\infty$, and $k=0,1,2, \ldots$ Furthermore, define the restricted germ-grain model

$$
Z(B)=\bigcup_{i: X_{i} \in B}\left(X_{i}+Z_{i}\right)
$$

The following lemma will serve as a cornerstone for the later proof of the asymptotic normality of $\eta(f, W)$.

Lemma 5.1. For any two pairs of bounded Borel sets $F, G$ and $\tilde{F}, \tilde{G}$ such that $F \subseteq \tilde{F}$, $G \subseteq \tilde{G}$ and $\tilde{F} \cap \tilde{G}=\emptyset$ we have

$$
\begin{aligned}
\beta\left(\mathfrak{A}_{\eta(f)}(F), \mathfrak{A}_{\eta(f)}(G)\right) \leq \beta\left(\mathfrak{A}_{\Psi}(\tilde{F}), \mathfrak{A}_{\Psi}(\tilde{G})\right) \\
+2 \mathbf{P}\left\{Z\left(\tilde{F}^{c}\right) \cap F \neq \emptyset\right\}+2 \mathbf{P}\left\{Z\left(\tilde{G}^{c}\right) \cap G \neq \emptyset\right\} .
\end{aligned}
$$

Remark 3. Estimate (5.3) is in the spirit of Mase [15] who derived upper bounds of the $\alpha$-mixing coefficient of germ-grain models. Explicit estimates of the $\beta$-mixing coefficient of Voronoi tessellations and Poisson cluster processes are given in [13].

Proof. We define the following dissections of $\Omega$ :

$$
\begin{aligned}
A_{k_{1}, \ldots, k_{p}} & =\left\{\eta\left(f, F_{1}\right) \in I_{k_{1}}^{(1)}, \ldots, \eta\left(f, F_{p}\right) \in I_{k_{p}}^{(p)}\right\} \\
B_{l_{1}, \ldots, l_{q}} & =\left\{\eta\left(f, G_{1}\right) \in J_{l_{1}}^{(1)}, \ldots, \eta\left(f, G_{q}\right) \in J_{l_{q}}^{(q)}\right\}
\end{aligned}
$$


for $k_{i}=1, \ldots, m_{i}, i=1, \ldots, p$, and $l_{j}=1, \ldots, n_{j}, j=1, \ldots, q$. Here the intervals

$$
I_{k_{i}}^{(i)}, k_{i}=1, \ldots, m_{i}, \quad\left(\operatorname{resp} . J_{l_{j}}^{(j)}, l_{j}=1, \ldots, n_{j}\right)
$$

are pairwise disjoint and

$$
I_{1}^{(1)} \cup \cdots \cup I_{m_{i}}^{(i)}=[0, \infty) \quad\left(\text { resp. } J_{1}^{(1)} \cup \cdots \cup J_{n_{j}}^{(j)}=[0, \infty)\right)
$$

for any $i=1, \ldots, p$ (resp. $j=1, \ldots, q$ ). Further, the Borel sets $F_{1}, \ldots, F_{p}$ (resp. $\left.G_{1}, \ldots, G_{q}\right)$ form an arbitrary partition of $F$ (resp. $G$ ). To simplify the writing, put

$$
A_{k}=A_{k_{1}, \ldots, k_{p}} \quad \text { and } \quad B_{l}=B_{l_{1}, \ldots, l_{q}}
$$

with the multiindices $k=\left(k_{1}, \ldots, k_{p}\right)$ and $l=\left(l_{1}, \ldots, l_{q}\right)$. According to the definition of the absolute regularity coefficient $\beta\left(\mathfrak{A}_{\eta(f)}(F), \mathfrak{A}_{\eta(f)}(G)\right)$ we have

$$
\beta\left(\mathfrak{A}_{\eta(f)}(F), \mathfrak{A}_{\eta(f)}(G)\right)=\frac{1}{2} \sup \sum_{k} \sum_{l}\left|\mathbf{P}\left(A_{k} \cap B_{l}\right)-\mathbf{P}\left(A_{k}\right) \mathbf{P}\left(B_{l}\right)\right|,
$$

where supremum stretches over all partitions $F_{1}, \ldots, F_{p}, G_{1}, \ldots, G_{q}, I_{1}^{(i)}, \ldots, I_{m_{i}}^{(i)}(i=$ $1, \ldots, p)$ and $J_{1}^{(j)}, \ldots, J_{n_{j}}^{(j)}(j=1, \ldots, q)$. We compare the events $A_{k}$ and $B_{l}$ with the corresponding events arising from the "truncated" random measure $\tilde{\eta}(f, \cdot)$ given by

$$
\tilde{\eta}(f, \cdot)=\sum_{i: i \geq 1} \mathbf{1}_{\tilde{F}}\left(X_{i}\right) \int_{\mathbb{U}} f(u) H_{Z_{i}}\left(d u,(\cdot) \backslash \Xi_{i}\right) .
$$

The very definition of $\tilde{\eta}(f, \cdot)$ and property $(3.3)$ of $H_{K}(\cdot)$ reveal that

$$
\left\{Z\left(\tilde{F}^{c}\right) \cap F=\emptyset\right\} \cap \tilde{A}_{k} \subseteq A_{k}
$$

and

$$
\left\{Z\left(\tilde{F}^{c}\right) \cap F=\emptyset\right\} \cap A_{k} \subseteq \tilde{A}_{k}
$$

where

$$
\tilde{A}_{k}=\left\{\tilde{\eta}\left(f, F_{1}\right) \in I_{k_{1}}^{(1)}, \ldots, \tilde{\eta}\left(f, F_{p}\right) \in I_{k_{p}}^{(p)}\right\} .
$$

Remember that $\eta\left(f, F^{*}\right)=\tilde{\eta}\left(f, F^{*}\right), F^{*} \subseteq F$, as soon as $Z\left(\tilde{F}^{c}\right) \cap F=\emptyset$. Likewise,

$$
\left\{Z\left(\tilde{G}^{c}\right) \cap G=\emptyset\right\} \cap \tilde{B}_{l} \subseteq B_{l} \quad \text { and } \quad\left\{Z\left(\tilde{G}^{c}\right) \cap G=\emptyset\right\} \cap B_{l} \subseteq \tilde{B}_{l} \text {, }
$$

where

$$
\tilde{B}_{l}=\left\{\tilde{\eta}\left(f, G_{1}\right) \in J_{l_{1}}^{(1)}, \ldots, \tilde{\eta}\left(f, G_{q}\right) \in J_{l_{q}}^{(q)}\right\} .
$$

From (5.4) and (5.5) it follows that

$$
\begin{aligned}
& \tilde{A}_{k} \cap A_{k}^{c} \subseteq\left\{Z\left(\tilde{F}^{c}\right) \cap F \neq \emptyset\right\} \cap \tilde{A}_{k}, \\
& A_{k} \cap \tilde{A}_{k}^{c} \subseteq\left\{Z\left(\tilde{F}^{c}\right) \cap F \neq \emptyset\right\} \cap A_{k} .
\end{aligned}
$$

Hence (with the abbreviation $A \triangle B=\left(A \cap B^{c}\right) \cup\left(A^{c} \cap B\right)$ )

$$
A_{k} \triangle \tilde{A}_{k} \subseteq\left\{Z\left(\tilde{F}^{c}\right) \cap F \neq \emptyset\right\} \cap\left(A_{k} \cup \tilde{A}_{k}\right)
$$


so that

$$
\sum_{k} \mathbf{P}\left(A_{k} \triangle \tilde{A}_{k}\right) \leq 2 \mathbf{P}\left\{Z\left(\tilde{F}^{c}\right) \cap F \neq \emptyset\right\}
$$

Analogously,

$$
\sum_{l} \mathbf{P}\left(B_{l} \triangle \tilde{B}_{l}\right) \leq 2 \mathbf{P}\left\{Z\left(\tilde{G}^{c}\right) \cap G \neq \emptyset\right\}
$$

After some elementary manipulations we get

$$
\begin{aligned}
\sum_{k} \sum_{l} \mid \mathbf{P}\left(A_{k} \cap\right. & \left.B_{l}\right)-\mathbf{P}\left(A_{k}\right) \mathbf{P}\left(B_{l}\right)-\left(\mathbf{P}\left(\tilde{A}_{k} \cap \tilde{B}_{l}\right)-\mathbf{P}\left(\tilde{A}_{k}\right) \mathbf{P}\left(\tilde{B}_{l}\right)\right) \mid \\
& \leq 2 \sum_{k} \mathbf{P}\left(A_{k} \triangle \tilde{A}_{k}\right)+2 \sum_{l} \mathbf{P}\left(B_{l} \triangle \tilde{B}_{l}\right) \\
& \leq 4 \mathbf{P}\left\{Z\left(\tilde{F}^{c}\right) \cap F \neq \emptyset\right\}+4 \mathbf{P}\left\{Z\left(\tilde{G}^{c}\right) \cap G \neq \emptyset\right\}
\end{aligned}
$$

By the assumed conditional independence within the sequence of grains (for given $\left.\Psi=\psi=\sum_{i: i \geq 1} \delta_{x_{i}}\right)$ and the construction of the truncated random measures $\tilde{\eta}\left(f, F^{\prime}\right)$, $\tilde{\eta}\left(f, G^{\prime}\right), F^{\prime} \subseteq F, G^{\prime} \subseteq G$, we can write

$$
\begin{aligned}
\mathbf{P}\left(\tilde{A}_{k} \cap \tilde{B}_{l}\right) & =\mathbf{E} \mathbf{P}\left(\tilde{A}_{k} \mid \mathfrak{A}_{\Psi}(\tilde{F})\right) \mathbf{P}\left(\tilde{B}_{k} \mid \mathfrak{A}_{\Psi}(\tilde{G})\right) \\
& =\int_{\Omega \times \Omega} \mathbf{P}\left(\tilde{A}_{k} \mid \mathfrak{A}_{\Psi}(\tilde{F})\right)\left(\omega_{1}\right) \mathbf{P}\left(\tilde{B}_{l} \mid \mathfrak{A}_{\Psi}(\tilde{G})\right)\left(\omega_{2}\right) \mathbf{P}_{\mathfrak{A}_{\Psi}(\tilde{F}) \otimes \mathfrak{A}_{\Psi}(\tilde{G})}\left(d\left(\omega_{1}, \omega_{2}\right)\right) .
\end{aligned}
$$

Together with the total probability rule

$$
\begin{aligned}
\mathbf{P}\left(\tilde{A}_{k}\right) & =\int_{\Omega} \mathbf{P}\left(\tilde{A}_{k} \mid \mathfrak{A}_{\Psi}(\tilde{F})\right)\left(\omega_{1}\right) \mathbf{P}_{\mathfrak{A}_{\Psi}(\tilde{F})}\left(d \omega_{1}\right), \\
\mathbf{P}\left(\tilde{B}_{l}\right) & =\int_{\Omega} \mathbf{P}\left(\tilde{B}_{l} \mid \mathfrak{A}_{\Psi}(\tilde{G})\right)\left(\omega_{2}\right) \mathbf{P}_{\mathfrak{A}_{\Psi}(\tilde{G})}\left(d \omega_{2}\right) .
\end{aligned}
$$

The double sum $\sum_{k} \sum_{l}\left|\mathbf{P}\left(\tilde{A}_{k} \cap \tilde{B}_{l}\right)-\mathbf{P}\left(\tilde{A}_{k}\right) \mathbf{P}\left(\tilde{B}_{l}\right)\right|$ can be written as

$$
\begin{aligned}
& \sum_{k} \sum_{l} \mid \int_{\Omega \times \Omega} \mathbf{P}\left(\tilde{A}_{k} \mid \mathfrak{A}_{\Psi}(\tilde{F})\right)\left(\omega_{1}\right) \mathbf{P}\left(\tilde{B}_{l} \mid \mathfrak{A}_{\Psi}(\tilde{G})\right)\left(\omega_{2}\right) \\
& \times\left(\mathbf{P}_{\mathfrak{A}_{\Psi}(\tilde{F}) \otimes \mathfrak{A}_{\Psi}(\tilde{G})}-\mathbf{P}_{\mathfrak{A}_{\Psi}(\tilde{F})} \times \mathbf{P}_{\mathfrak{A}_{\Psi}(\tilde{G})}\right)\left(d\left(\omega_{1}, \omega_{2}\right)\right) \mid \\
& \leq \int_{\Omega \times \Omega}\left|\mathbf{P}_{\mathfrak{A}_{\Psi}(\tilde{F}) \otimes \mathfrak{A}_{\Psi}(\tilde{G})}-\mathbf{P}_{\mathfrak{A}_{\Psi}(\tilde{F})} \times \mathbf{P}_{\mathfrak{A}_{\Psi}(\tilde{G})}\right|\left(d\left(\omega_{1}, \omega_{2}\right)\right)=2 \beta\left(\mathfrak{A}_{\Psi}(\tilde{F}), \mathfrak{A}_{\Psi}(\tilde{G})\right) .
\end{aligned}
$$

The latter estimate combined with (5.6) completes the proof of Lemma 5.1.

Next we specify (5.3) for

$$
\begin{array}{cl}
F=[-a, a]^{d} & , \quad G=\mathbb{R}^{d} \backslash[-b, b]^{d}, \\
\tilde{F}=[-(a+\Delta), a+\Delta]^{d}, & \tilde{G}=\mathbb{R}^{d} \backslash[-(b-\Delta), b-\Delta]^{d}
\end{array}
$$

with $0<a<b<\infty$ and $\Delta=(b-a) / 4$. Note that by a simple approximation argument inequality (5.3) remains valid for unbounded $G$ and $\tilde{G}$. 
Lemma 5.2. Let $\Psi=\sum_{i: i \geq 1} \delta_{X_{i}}$ be a stationary point process with intensity $\lambda>0$. Assume that the typical grain $Z_{0}$ is a.s. compact such that

$$
\mathbf{E}\left\|Z_{0}\right\|^{d}<\infty \text {. }
$$

Then

$$
\mathbf{P}\left\{Z\left(\tilde{F}^{c}\right) \cap F \neq \emptyset\right\} \leq \lambda d 2^{d}\left(1+\frac{a}{\Delta}\right)^{d-1} \int_{\Delta}^{\infty} x^{d} d D(x)
$$

and

$$
\mathbf{P}\left\{Z\left(\tilde{G}^{c}\right) \cap G \neq \emptyset\right\} \leq \lambda d 2^{d}\left(1+\frac{3 a}{\Delta}\right)^{d-1} \int_{\Delta}^{\infty} x^{d} d D(x),
$$

where $D(x)=\mathbf{P}\left\{\left\|Z_{0}\right\| \leq x\right\}, x \geq 0$.

ProOF. By the definition of the germ-grain model (3.1) we obtain that

$$
\mathbf{P}\left\{Z\left(\tilde{F}^{c}\right) \cap F \neq \emptyset\right\}=\mathbf{E}\left[\prod_{i: i \geq 1}\left(1-\left(1-\mathbf{1}_{\tilde{F}}\left(X_{i}\right)\right) \mathbf{P}\left\{\left(Z_{0}+X_{i}\right) \cap F \neq \emptyset \mid \Psi\right\}\right)\right],
$$

where $\mathbf{P}\{\cdot \mid \Psi\}$ is the conditional probability with respect to $\Psi$. Using the elementary inequality $1-\Pi\left(1-x_{i}\right) \leq \sum x_{i}, 0 \leq x_{i} \leq 1$, and applying Campbell's and Fubini's theorem lead to

$$
\begin{aligned}
\mathbf{P}\left\{Z\left(\tilde{F}^{c}\right) \cap F \neq \emptyset\right\} & \leq \lambda \int_{\tilde{F}^{c}} \mathbf{P}\left\{x \in \check{Z}_{0} \oplus F\right\} d x \\
& =\lambda \mathbf{E}\left|\tilde{F}^{c} \cap\left(F \oplus \check{Z}_{0}\right)\right| .
\end{aligned}
$$

Since

$$
\check{Z}_{0} \oplus F \subseteq\left[-\left(a+\left\|Z_{0}\right\|\right), a+\left\|Z_{0}\right\|\right]^{d}
$$

we may continue with

$$
\begin{aligned}
\mathbf{P}\left\{Z\left(\tilde{F}^{c}\right) \cap F \neq \emptyset\right\} & \leq \lambda \mathbf{E}\left|\left([-(a+\Delta), a+\Delta]^{d}\right)^{c} \backslash\left[-\left(a+\left\|Z_{0}\right\|\right), a+\left\|Z_{0}\right\|\right]\right| \\
& =\lambda 2^{d} \int_{\Delta}^{\infty}\left((a+x)^{d}-(a+\Delta)^{d}\right) d D(x) \\
& \leq \lambda d 2^{d}\left(\frac{a+\Delta}{\Delta}\right)^{d-1} \int_{\Delta}^{\infty} x^{d} d D(x) .
\end{aligned}
$$

In a similar way we treat probability (5.9) and get

$$
\begin{aligned}
\mathbf{P}\left\{Z\left(\tilde{G}^{c}\right) \cap G \neq \emptyset\right\} & \leq \lambda \mathbf{E}\left|[-(b-\Delta), b-\Delta]^{d} \backslash\left(\left[-\left(b-\left\|Z_{0}\right\|\right), b-\left\|Z_{0}\right\|\right]\right)^{c}\right| \\
& =\lambda 2^{d} \int_{\Delta}^{b}\left((b-\Delta)^{d}-(b-x)^{d}\right) d D(x) \\
& \leq \lambda d 2^{d}\left(\frac{b-\Delta}{\Delta}\right)^{d-1} \int_{\Delta}^{\infty} x^{d} d D(x) .
\end{aligned}
$$

Thus, by $b-\Delta=a+3 \Delta$, the proof of Lemma 5.2 is completed. 


\section{Limit Theorems for Associated Random Measures}

The ergodic theorem of Nguyen and Zessin [19] can be used to establish the spatial strong law of large numbers for the measure $\eta(\Gamma, W)$ provided $\eta(\Gamma, W)$ is ergodic and $W$ expands infinitely in all directions in a regular way. The latter is denoted by $W \uparrow$ $\mathbb{R}^{d}$ and means that all $W$ 's are convex, compact, increasing and $r(W)=\sup \{r>0$ : $W$ contains a ball of radius $r\} \rightarrow \infty$, see [4, p. 332].

Theorem 6.1. In addition to the basic assumptions suppose that $\Psi$ is ergodic (under $d$-dimensional shifts, see [4, p. 341]). Then, for any $\Gamma \in \mathfrak{U}$,

$$
|W|^{-1} \eta(\Gamma, W) \rightarrow \lambda(\Gamma) \quad \text { a.s. as } \quad W \uparrow \mathbb{R}^{d}
$$

with $\lambda(\Gamma)$ as defined in Lemma 4.1.

Proof. First, note that for any $K \in \mathcal{K}$ we have

$$
\int_{\mathbb{R}^{d}} H_{K}(\Gamma, W+x) d x=|W| H_{K}\left(\Gamma, \mathbb{R}^{d}\right) .
$$

In [11] it was proved that the ergodicity of $\Psi$ entails the ergodicity of $Z$ provided (3.2) is valid. This, in turn, implies that the spatial stochastic process $\eta(\Gamma, F), F \in \mathfrak{B}_{0}^{d}$, ( $\Gamma$ is fixed) is ergodic under $d$-dimensional shifts. In order to apply Corollary 4.20 in [19], it is necessary to bound the family $\eta(\Gamma, F), F \subseteq[0,1)^{d}, F \in \mathfrak{B}_{0}^{d}$, by some integrable random variable $Y$ being independent of $F$. By (3.3) and (3.4),

$$
\eta(\Gamma, F) \leq \sum_{i: i \geq 1} H_{Z_{i}}\left(\Gamma,[0,1)^{d}-X_{i}\right)=Y .
$$

Together with Campbell's theorem,

$$
\mathbf{E} Y \leq \lambda \int_{\mathbb{R}^{d}} \mathbf{E} H_{Z_{0}+x}\left(\mathbb{U},[0,1)^{d}\right) d x=\lambda \bar{H}\left(\Gamma, \mathbb{R}^{d}\right)<\infty
$$

proving (6.1).

This theorem, in particular, yields the almost sure convergence of spatial intensities for extensions of the intrinsic volumes, see also [11] and [27]. The results about uniform convergence for $W$ belonging to a certain class of sets $\mathcal{W}$ can be obtained from the general theory presented in [3].

Below we formulate a central limit theorem for the finite-dimensional distributions of the set-indexed sequence

$$
\hat{\eta}\left(\Gamma, W_{n}\right)=\left|W_{n}\right|^{1 / 2}\left(\frac{\eta\left(\Gamma, W_{n}\right)}{\left|W_{n}\right|}-\lambda(\Gamma)\right), \quad \Gamma \in \mathfrak{U},
$$


where $W_{n}$ denotes the cube $[-n, n)^{d}$. For doing this, we need a suitable formulation of the $\beta$-mixing condition imposed on the underlying point process $\Psi=\sum_{i: i \geq 1} \delta_{X_{i}}$. Assume that

$$
\beta\left(\mathfrak{A}_{\Psi}\left([-a, a]^{d}\right), \mathfrak{A}_{\Psi}\left(\mathbb{R}^{d} \backslash[-(a+\Delta), a+\Delta]^{d}\right)\right) \leq\left(\frac{a}{\min (a, \Delta)}\right)^{d-1} \beta_{\Psi}(\Delta)
$$

for any $a, \Delta \geq 1$, where $\beta_{\Psi}(\cdot)$ is a non-decreasing function on $[1, \infty)$.

Theorem 6.2. In addition to the above basic assumptions suppose that there exists $\delta>0$ such that

$$
\begin{aligned}
\mathbf{E}\left[\sum_{i: i \geq 1} H_{X_{i}+Z_{i}}\left(\mathbb{U},[0,1)^{d}\right)\right]^{2+\delta} & <\infty \\
\mathbf{E}\left\|Z_{0}\right\|^{2 d(1+\delta) / \delta+\varepsilon} & <\infty \text { for some } \varepsilon>0 \\
\sum_{n=1}^{\infty} n^{d-1}\left(\beta_{\Psi}(n)\right)^{\delta /(2+\delta)} & <\infty
\end{aligned}
$$

(If

$$
\mathbf{P}\left\{\sum_{i: i \geq 1} H_{X_{i}+Z_{i}}\left(\mathbb{U},[0,1)^{d}\right) \leq c\right\}=1
$$

for some constant $c<\infty$, then put $\delta=\infty$ and $\varepsilon=0$ in (6.6) and (6.7).)

Then, for any $k$-tuple $\Gamma_{1}, \ldots, \Gamma_{k} \in \mathfrak{U}$, the random vector $\left(\hat{\eta}\left(\Gamma_{1}, W_{n}\right), \ldots, \hat{\eta}\left(\Gamma_{k}, W_{n}\right)\right)$ converges in distribution as $n \rightarrow \infty$ to a $k$-dimensional centred Gaussian random vector $\left(\xi_{1}, \ldots, \xi_{k}\right)$ with covariances $\mathbf{E} \xi_{i} \xi_{j}=\sigma^{2}\left(\Gamma_{i}, \Gamma_{j}\right)$ defined by

$$
\begin{aligned}
& \sigma^{2}\left(\Gamma_{i}, \Gamma_{j}\right) \\
&=\lambda \int_{\left(\mathbb{R}^{d}\right)^{2}} G_{0}^{!} {\left[\mathbf{P}\left\{(\cdot) \notin\left(\check{Z}_{0}+y_{1}\right) \cup\left(\check{Z}_{0}+y_{2}\right)\right\}\right] \bar{H}\left(\Gamma_{i}, d y_{1} ; \Gamma_{j}, d y_{2}\right) } \\
&+\lambda \int_{\mathbb{R}^{d}\left(\mathbb{R}^{d}\right)^{2}} G_{0, x}^{!}\left[\mathbf{P}\left\{(\cdot) \notin\left(\check{Z}_{0}+y_{1}\right) \cup\left(\check{Z}_{0}+x+y_{2}\right)\right\}\right] \\
& \times \\
& \times \Phi\left(x ; \Gamma_{i}, d y_{1} ; \Gamma_{j}, d y_{2}\right) \gamma_{\mathrm{red}}^{(2)}(d x) \\
&+\lambda \int_{\mathbb{R}^{d}\left(\mathbb{R}^{d}\right)^{2}}\left[G_{0, x+y_{1}-y_{2}}^{!}\left[\mathbf{P}\left\{(\cdot) \notin\left(\check{Z}_{0}+y_{1}\right) \cup\left(\check{Z}_{0}+x+y_{1}\right)\right\}\right]\right. \\
&\left.-G_{0}^{!}\left[\mathbf{P}\left\{(\cdot) \notin \check{Z}_{0}+y_{1}\right\}\right] G_{0}^{!}\left[\mathbf{P}\left\{(\cdot) \notin \check{Z}_{0}+y_{2}\right\}\right]\right] \\
& \times \Phi\left(x, \Gamma_{i}, d y_{1}\right) \Phi\left(-x, \Gamma_{j}, d y_{2}\right) d x \\
&-\lambda^{2} \int_{\mathbb{R}^{d}\left(\mathbb{R}^{d}\right)^{2}} G_{0}^{!}\left[\mathbf{P}\left\{(\cdot) \notin\left(\check{Z}_{0}+y_{1}\right)\right\}\right] G_{0}^{!}\left[\mathbf{P}\left\{(\cdot) \notin\left(\check{Z}_{0}+y_{2}\right)\right\}\right] \\
& \times \tilde{\Phi}\left(x, \Gamma_{i}, d y_{1}\right) \tilde{\Phi}\left(-x, \Gamma_{j}, d y_{2}\right) d x, \quad 1 \leq i \leq j \leq k,
\end{aligned}
$$


where $\bar{H}\left(\Gamma_{i}, d y_{1} ; \Gamma_{j}, d y_{2}\right)$ and $\Phi\left(x ; \Gamma_{i}, d y_{1} ; \Gamma_{j}, d y_{2}\right)$ have been defined in (4.7) and (4.8) respectively, and

$$
\begin{aligned}
\Phi(x, \Gamma, W) & =\mathbf{E}\left[\int_{W}\left(1-\mathbf{1}_{Z_{0}}(y-x)\right) H_{Z_{0}}(\Gamma, d y)\right] \\
& \left.=\mathbf{E}\left[H_{Z_{0}}\left(\Gamma,\left(Z_{0}^{c}+x\right) \cap W\right)\right)\right], \\
\tilde{\Phi}(x, \Gamma, W) & =\bar{H}(\Gamma, W)-\Phi(x, \Gamma, W) .
\end{aligned}
$$

In (6.8) all integrals converge absolutely as a consequence of the mixing and moment conditions (6.5)-(6.7).

Remark 4. Let $l$ denote the smallest integer greater than or equal to $2+\delta$. Then the conditions

$$
\operatorname{Var}\left(\gamma_{\text {red }}^{(k)}\right)<\infty, \quad k=2, \ldots, l, \quad \text { and } \quad \mathbf{E} H_{Z_{0}}^{l}\left(\mathbb{U}, \mathbb{R}^{d}\right)<\infty
$$

suffice to hold (6.5). This is immediately seen from Lemma 4.2.

Remark 5. The $\beta$-mixing condition (6.7) can be verified for quite a few classes of point processes under mild additional assumptions. For example, in the special case of a Poisson cluster process $\Psi$, that is, the support of $\Psi$ is a Boolean model with a random discrete a.s. finite typical grain (cluster) $Z_{c}$, we have by Lemmata 5.1 and 5.2 that

$$
\beta_{\Psi}(t) \leq 4 \lambda d 6^{d-1} \mathbf{E} \rho_{c}^{d} \mathbf{1}_{\left\{\rho_{c} \geq t / 2\right\}}
$$

with $\rho_{c}=\sup \left\{\|x\|: x \in Z_{c}\right\}$.

Similar estimates of the $\beta$-mixing rate are known for

- dependently thinned (Poisson) point processes (e.g., soft- and hard-core processes) as defined by Matèrn and their generalizations, see [23];

- Gibbsian point processes satisfying Dobrushin's uniqueness conditions;

- point processes generated by a (Poisson-) Voronoi tessellation of $\mathbb{R}^{d}$ (e.g., vertices, midpoint of edges), see [13].

Proof of Theorem 6.2. According to the well-known Cramér-Wold device we need to prove that, for any $\left(a_{1}, \ldots, a_{k}\right) \in \mathbb{R}^{k} \backslash\{0\}$, the sum

$$
S_{n}=\sum_{j=1}^{k} a_{j} \hat{\eta}\left(\Gamma_{j}, W_{n}\right)
$$

converges weakly as $n \rightarrow \infty$ to a Gaussian random variable $\xi$ with mean zero and variance $\sigma^{2}=\sum_{i, j=1}^{k} a_{i} a_{j} \sigma^{2}\left(\Gamma_{i}, \Gamma_{j}\right)$. In order to apply a central limit theorem for stationary $\beta$ mixing random fields in [13] we rewrite the above sum as

$$
\left|W_{n}\right|^{1 / 2} S_{n}=\sum_{z \in I_{n}} X_{z}, \quad X_{z}=\sum_{j=1}^{k} a_{j}\left(\eta\left(\Gamma_{j}, E_{z}\right)-\lambda\left(\Gamma_{j}\right)\right)
$$


$E_{z}=[0,1)^{d}+z$ with $z \in I_{n}=\{-n, \ldots, 0, \ldots, n-1\}^{d}$.

From the simple estimate

$$
\eta\left(\mathbb{U},[0,1)^{d}\right) \leq \sum_{i: i \geq 1} H_{Z_{i}+X_{i}}\left(\mathbb{U},[0,1)^{d}\right)
$$

together with (6.5) we deduce that $\mathbf{E}\left|X_{0}\right|^{2+\delta}<\infty$. In view of Lemmata 5.1 and 5.2 combined with (6.4) we obtain the following estimate

$$
\begin{aligned}
& \beta\left(\mathfrak{A}_{\eta(f)}\left([-a, a]^{d}\right), \mathfrak{A}_{\eta(f)}\left(\mathbb{R}^{d} \backslash[-(a+\Delta), a+\Delta]^{d}\right)\right) \\
& \quad \leq\left(\frac{4 a+\Delta}{2 \Delta}\right)^{d-1} \beta_{\Psi}(\Delta / 2)+\lambda d 2^{d+1} \int_{\Delta / 4}^{\infty} x^{d} d D(x)\left[\left(\frac{4 a+\Delta}{\Delta}\right)^{d-1}+\left(\frac{12 a+\Delta}{\Delta}\right)^{d-1}\right]
\end{aligned}
$$

for all $a, \Delta \geq 1$ and any bounded measurable $f: \mathbb{U} \mapsto[0, \infty)$. The right-hand side of the latter inequality can be bounded by a term of the form

$$
\left(\frac{a}{\min (a, \Delta)}\right)^{d-1} \beta_{\eta}(\Delta)
$$

with

$$
\beta_{\eta}(\Delta)=c_{1} \beta_{\Psi}(\Delta / 2)+c_{2} \int_{\Delta / 4}^{\infty} x^{d} d D(x)
$$

and constants $c_{1}$ and $c_{2}$ depending only on $d$.

Finally, in order to prove the $\beta$-mixing condition needed in the central limit theorem in [13] we have to ensure that

$$
\sum_{n=1}^{\infty} n^{d-1}\left(\beta_{\eta}(n)\right)^{\delta /(2+\delta)}<\infty \quad \text { and } \quad n^{d} \beta_{\eta}(n) \rightarrow 0 \quad \text { as } \quad n \rightarrow \infty .
$$

In turn, this follows from (6.6), (6.7) and the fact that $\beta_{\eta}(\cdot)$ is a non-decreasing function.

If $\left|X_{z}\right| \leq c, z \in I_{n}$, for some constant $c$, we need the convergence of the series

$$
\sum_{n=1}^{\infty} n^{d-1} \beta_{\eta}(n)
$$

and

$$
\sum_{n=1}^{\infty} n^{d-1} \beta_{\Psi}(n / 2)+\sum_{n=1}^{\infty} n^{d-1} \int_{n / 4}^{\infty} x^{d} d D(x) .
$$

This results from (6.7) for $\delta=\infty$ and $\mathbf{E}\left\|Z_{0}\right\|^{2 d}<\infty$, so that the proof of Theorem 6.2 is completed. 
7. A Central Limit Theorem for Random Measures Generated by Boolean MODELS

From now on, let $\Psi$ be a stationary Poisson process with intensity $\lambda>0$, i.e., the corresponding germ-grain model $Z$ is a Boolean model $[16,23]$. In this case, by Slivnyak's theorem, $\gamma_{\text {red }}^{(2)}(\cdot)$ vanishes identically and

$$
\begin{aligned}
G_{x_{1}, x_{2}}^{!}\left[\mathbf{P}\left\{(\cdot) \notin\left(\check{Z}_{0} \cup\left(\check{Z}_{0}+v\right)\right)\right\}\right] & =\exp \left\{-\lambda \mathbf{E}\left|\check{Z}_{0} \cup\left(\check{Z}_{0}+v\right)\right|\right\} \\
& =\exp \left\{-\lambda \mathbf{E}\left|Z_{0} \cup\left(Z_{0}+v\right)\right|\right\} \\
& =q(v)(1-p)^{2},
\end{aligned}
$$

where

$$
p=\mathbf{P}\{0 \in Z\}=1-\exp \left\{-\lambda \mathbf{E}\left|Z_{0}\right|\right\}
$$

is the volume fraction of $Z$,

$$
q(v)=\frac{C(v)-p^{2}}{(1-p)^{2}}+1=\exp \left\{\lambda \mathbf{E}\left|Z_{0} \cap\left(Z_{0}+v\right)\right|\right\}, \quad v \in \mathbb{R}^{d},
$$

and $C(v)=\mathbf{P}\{0 \in Z, v \in Z\}$ is the covariance function of $Z$.

Using these formulae together with (6.9) we can simplify the covariances $\sigma^{2}\left(\Gamma_{i}, \Gamma_{j}\right)$ in Theorem 6.2 as follows:

$$
\begin{aligned}
\sigma^{2}\left(\Gamma_{i}, \Gamma_{j}\right) & =\lambda(1-p)^{2} \int_{\mathbb{R}^{d}} \int_{\mathbb{R}^{d}} q\left(y_{1}-y_{2}\right) \bar{H}\left(\Gamma_{i}, d y_{1} ; \Gamma_{j}, d y_{2}\right) \\
& +\lambda^{2}(1-p)^{2} \int_{\mathbb{R}^{d}}\left(q(x) \Phi\left(x, \Gamma_{i}\right) \Phi\left(-x, \Gamma_{j}\right)-\bar{H}\left(\Gamma_{i}, \mathbb{R}^{d}\right) \bar{H}\left(\Gamma_{j}, \mathbb{R}^{d}\right)\right) d x,
\end{aligned}
$$

where

$$
\left.\Phi(x, \Gamma)=\Phi\left(x, \Gamma, \mathbb{R}^{d}\right)=\mathbf{E}\left[H_{Z_{0}}\left(\Gamma, Z_{0}^{c}+x\right)\right)\right] .
$$

Taking into account the inequalities

$$
\int_{\mathbb{R}^{d}}(q(x)-1) d x \leq \lambda \mathbf{E}\left|Z_{0}\right|^{2} \exp \left\{\lambda \mathbf{E}\left|Z_{0}\right|\right\}
$$

and

$$
\begin{aligned}
\int_{\mathbb{R}^{d}}\left|\Phi\left(x, \Gamma_{i}\right) \Phi\left(-x, \Gamma_{j}\right)-\bar{H}\left(\Gamma_{i}, \mathbb{R}^{d}\right) \bar{H}\left(\Gamma_{j}, \mathbb{R}^{d}\right)\right| d x & \\
& \leq \mathbf{E}\left|Z_{0}\right| H_{Z_{0}}\left(\Gamma_{i}, \mathbb{R}^{d}\right)+\mathbf{E}\left|Z_{0}\right| H_{Z_{0}}\left(\Gamma_{j}, \mathbb{R}^{d}\right),
\end{aligned}
$$

we conclude that $\sigma^{2}\left(\Gamma_{i}, \Gamma_{j}\right), 1 \leq i, j \leq k$, are finite whenever

$$
\mathbf{E}\left|Z_{0}\right|^{2}<\infty \quad \text { and } \quad \mathbf{E} H_{Z_{0}}^{2}\left(\Gamma_{i}, \mathbb{R}^{d}\right)<\infty \quad \text { for } \quad i=1, \ldots, k .
$$

The following Theorem 7.1 restates the main result of the preceding section in case of a stationary Poisson process of germs under considerably relaxed conditions. In fact, these conditions are optimal because they are necessary to ensure the existence of the covariance matrix. This improvement results from a suitable (although somewhat laborious) approximation technique by $m$-dependent fields, which is quite different from that used in $[9,10]$ and $[12]$. 
Theorem 7.1. If $\Psi$ is a stationary Poisson process with intensity $\lambda$ and (7.4) is satisfied, then $\left(\hat{\eta}\left(\Gamma_{1}, W_{n}\right), \ldots, \hat{\eta}\left(\Gamma_{k}, W_{n}\right)\right)$ converges in distribution as $n \rightarrow \infty$ to a Gaussian centred random vector $\left(\xi_{1}, \ldots, \xi_{k}\right)$ with the covariances $\mathbf{E} \xi_{i} \xi_{j}=\sigma^{2}\left(\Gamma_{i} \Gamma_{j}\right), 1 \leq i, j \leq k$, given by (7.2).

Proof. We only need to consider the univariate case $k=1$ for some fixed $\Gamma=\Gamma_{1} \in \mathfrak{U}$. With the notations introduced in the proof of Theorem 6.2 we put

$$
S_{n}=\left|W_{n}\right|^{-1 / 2} \sum_{z \in I_{n}} X_{z} \quad \text { with } \quad X_{z}=\eta\left(\Gamma, E_{z}\right)-\lambda(1-p) \bar{H}\left(\Gamma, \mathbb{R}^{d}\right) .
$$

Setting $F_{z}=E_{z} \oplus W_{m}$ for some fixed integer $m \geq 1$ and $z \in \mathbb{Z}^{d}=\{0, \pm 1, \pm 2, \ldots\}^{d}$ we decompose $S_{n}=S_{n}^{(m)}+S_{n, 1}^{(m)}+S_{n, 2}^{(m)}$ by splitting $X_{z}$ into three random variables:

$$
\begin{aligned}
X_{z}^{(m)} & =\sum_{i: i \geq 1} \mathbf{1}_{F_{z}}\left(X_{i}\right) H_{Z_{i}+X_{i}}\left(\Gamma, E_{z} \backslash \Xi_{i}\left(F_{z}\right)\right) \quad \text { with } \quad \Xi_{i}(B)=\bigcup_{j: j \neq i, X_{j} \in B}\left(Z_{j}+X_{j}\right), \\
X_{z, 1}^{(m)} & =\sum_{i: i \geq 1} \mathbf{1}_{F_{z}^{c}}\left(X_{i}\right) H_{Z_{i}+X_{i}}\left(\Gamma, E_{z} \backslash \Xi_{i}\left(F_{z}\right)\right) \\
X_{z, 2}^{(m)} & =\sum_{i: i \geq 1}\left(H_{Z_{i}+X_{i}}\left(\Gamma, E_{z} \backslash \Xi_{i}\right)-H_{Z_{i}+X_{i}}\left(\Gamma, E_{z} \backslash \Xi_{i}\left(F_{z}\right)\right)\right) .
\end{aligned}
$$

We first note that, by our assumptions, the independently marked Poisson counting measures

$$
\Psi_{z}=\sum_{i: i \geq 1} \mathbf{1}_{E_{z}}\left(X_{i}\right) \delta_{\left[X_{i}, Z_{i}\right]}, \quad z \in \mathbb{Z}^{d},
$$

can be considered as a family of independent identically distributed random elements taking values in some measurable space $\left[N_{\text {mark }}, \mathfrak{N}_{\text {mark }}\right]$ of marked counting measures defined on $[0,1)^{d} \times \mathcal{K}$, see [5] for details. Therefore, having in mind the properties of $H_{K}(\cdot)$, $K \in \mathcal{K}$, it is easily seen that the random variables $X_{z}^{(m)}, z \in I_{n}$, constitute a stationary $2 m$-dependent random field which even allows a so-called block-representation

$$
X_{z}^{(m)}=g\left(\Psi_{y} ; y \in\{-m, \ldots, m\}^{d}+z\right), \quad z \in \mathbb{Z}^{d},
$$

where $g: N_{\text {mark }}^{(2 m+1)^{d}} \mapsto \mathbb{R}^{1}$ is some $\mathfrak{N}_{\text {mark }}^{(2 m+1)^{d}}$-measurable function. Applying the central limit theorem for this type of weakly dependent fields, see, e.g., Heinrich [9], yields the weak convergence of $S_{n}^{(m)}($ as $n \rightarrow \infty)$ to a centred Gaussian random variable with variance

$$
\sigma_{m}^{2}=\sum_{z \in\{-m, \ldots, m\}^{d}} \operatorname{Cov}\left(X_{0}^{(m)}, X_{z}^{(m)}\right)
$$

provided that $\mathbf{E}\left(X_{0}^{(m)}\right)^{2}<\infty$. The latter holds for any $m \geq 1$, if $\mathbf{E}\left|Z_{0}\right|^{2}<\infty$ and $\mathbf{E} H_{Z_{0}}^{2}\left(\Gamma, \mathbb{R}^{d}\right)<\infty$. In order to prove the asymptotic normality of $S_{n}$ it remains to verify that

$$
\sup _{n \geq 1} \mathbf{E}\left(S_{n, i}^{(m)}\right)^{2} \leq s_{i}^{(m)}=\sum_{z \in \mathbb{Z}^{d}}\left|\operatorname{Cov}\left(X_{0, i}^{(m)} X_{z, i}^{(m)}\right)\right| \longrightarrow 0 \quad \text { as } \quad m \rightarrow \infty .
$$

for $i=1,2$. 
After straightforward calculations similar to those leading to (7.2) followed by some obvious estimates we obtain

$$
\begin{aligned}
& \left|\operatorname{Cov}\left(X_{0,1}^{(m)}, X_{z, 1}^{(m)}\right)\right| \leq \lambda \int_{F_{0}^{c} \cap F_{z}^{c}} \mathbf{E}\left[H_{Z_{0}}\left(\Gamma, E_{0}-x\right) H_{Z_{0}}\left(\Gamma, E_{z}-x\right)\right] d x \\
& +\lambda^{2} \iint_{F_{0}^{c}} \int_{F_{z}^{c}} \int_{\mathcal{K}} \int_{\mathcal{K}} \int_{\mathbb{R}^{d}} \mathbf{1}_{\mathbb{R}^{d}}\left(x_{1}+y_{1}\right) \mathbf{1}_{E_{z}}\left(x_{2}+y_{2}\right)\left(a\left(\mathbb{R}^{d}, K_{2}\right)+b\left(\mathbb{R}^{d}, K_{1}\right)+\right. \\
& \left.\quad+1-\exp \left\{-w\left(F_{0}, F_{z}\right)\right\}\right) H_{K_{1}}\left(\Gamma, d y_{1}\right) H_{K_{2}}\left(\Gamma, d y_{2}\right) Q\left(d K_{1}\right) Q\left(d K_{2}\right) d x_{2} d x_{1},
\end{aligned}
$$

where

$$
a\left(F_{0}, K_{2}\right)=\mathbf{1}_{F_{0} \cap\left(\check{K}_{2}+x_{1}+y_{1}\right)}\left(x_{2}\right), \quad b\left(F_{z}, K_{1}\right)=\mathbf{1}_{F_{z} \cap\left(\check{K}_{1}+x_{2}+y_{2}\right)}\left(x_{1}\right),
$$

and

$$
w\left(F_{0}, F_{z}\right)=\lambda \mathbf{E}\left|F_{0} \cap\left(\check{Z}_{0}+x_{1}+y_{1}\right) \cap F_{z} \cap\left(\check{Z}_{0}+x_{2}+y_{2}\right)\right| .
$$

Therefore,

$$
\begin{aligned}
s_{1}^{(m)} \leq & \lambda \int_{F_{0}^{c}} \mathbf{E}\left[H_{Z_{0}}\left(\Gamma, E_{0}-x\right) H_{Z_{0}}\left(\Gamma, \mathbb{R}^{d}\right)\right] d x+2 \lambda^{2} \int_{F_{0}^{c}} \mathbf{E}\left[\left|Z_{0}\right| H_{Z_{0}}\left(\Gamma, E_{0}-x\right)\right] d x \\
& +\lambda^{3} \mathbf{E}\left|Z_{0}\right|^{2} \bar{H}\left(\Gamma, \mathbb{R}^{d}\right) \int_{F_{0}^{c}} \bar{H}\left(\Gamma, E_{0}-x\right) d x .
\end{aligned}
$$

In view of our moment assumptions (7.4), it follows from the Lebesgue dominated convergence theorem that the right-hand side tends to zero as $m \rightarrow \infty$.

Next we estimate the covariances occurring in $s_{2}^{(m)}$. For notational simplicity write

$$
u\left(F_{0}\right)=\lambda \mathbf{E}\left|\left(F_{0}-\left(x_{1}+y_{1}\right)\right) \cap \check{Z}_{0}\right|, \quad v\left(F_{z}\right)=\lambda \mathbf{E}\left|\left(F_{z}-\left(x_{2}+y_{2}\right)\right) \cap \check{Z}_{0}\right|,
$$

and

$$
\begin{aligned}
& I\left(F_{0}, F_{z}\right) \\
& \quad=\exp \left\{-\left(u\left(F_{0}\right)+v\left(F_{z}\right)\right)\right\}\left[\left(1-a\left(F_{0}, K_{2}\right)\right)\left(1-b\left(F_{z}, K_{1}\right)\right) \exp \left\{w\left(F_{0}, F_{z}\right)\right\}-1\right] .
\end{aligned}
$$

Then

$$
\begin{aligned}
& \left|\operatorname{Cov}\left(X_{0,2}^{(m)}, X_{z, 2}^{(m)}\right)\right| \leq \lambda \mathbf{E} \iint_{\mathbb{R}^{d}} \int_{\mathbb{R}^{d}} \mathbf{1}_{\mathbb{R}^{d}}\left(x+y_{1}\right) \mathbf{1}_{E_{z}}\left(x+y_{2}\right) \\
& \times\left(\exp \left\{-\lambda \mathbf{E}\left|\left(F_{0}-\left(x+y_{1}\right)\right) \cap \check{Z}_{0}\right|\right\}-\exp \left\{-\lambda \mathbf{E}\left|\check{Z}_{0}\right|\right\}\right) H_{Z_{0}}\left(\Gamma, d y_{1}\right) H_{Z_{0}}\left(\Gamma, d y_{2}\right) d x \\
& +\lambda^{2} \iint_{\mathbb{R}^{d}} \int_{\mathbb{R}^{d}} \iint_{\mathcal{K}} \int_{\mathbb{R}^{d}} \mathbf{1}_{\mathbb{R}^{d}}\left(x_{1}+y_{1}\right) \mathbf{1}_{E_{z}}\left(x_{2}+y_{2}\right) \mid I\left(\mathbb{R}^{d}, \mathbb{R}^{d}\right)-I\left(\mathbb{R}^{d}, F_{z}\right) \\
& \quad-I\left(F_{0}, \mathbb{R}^{d}\right)+I\left(F_{0}, F_{z}\right) \mid H_{K_{1}}\left(\Gamma, d y_{1}\right) H_{K_{2}}\left(\Gamma, d y_{2}\right) Q\left(d K_{1}\right) Q\left(d K_{2}\right) d x_{2} d x_{1} \\
& \leq \lambda^{2} \mathbf{E}\left|\left(F_{0}^{c} \oplus \check{E}_{0}\right) \cap \check{Z}_{0}\right| \int_{\mathbb{R}^{d}} \mathbf{E}\left[H_{Z_{0}}\left(\Gamma, E_{0}-x\right) H_{Z_{0}}\left(\Gamma, E_{z}-x\right)\right] d x
\end{aligned}
$$




$$
\begin{aligned}
& +\lambda^{2} \iint_{\mathbb{R}^{d}} \int_{\mathbb{R}^{d}} \int_{\mathcal{K}} \int_{\mathcal{K}} \int_{\mathbb{R}^{d}} \int_{\mathbb{R}^{d}} \mathbf{1}_{E_{0}}\left(x_{1}+y_{1}\right) \mathbf{1}_{E_{z}}\left(x_{2}+y_{2}\right)\left(u ( F _ { 0 } ^ { c } ) v ( F _ { z } ^ { c } ) \left[w\left(F_{0}, F_{z}\right)+a\left(F_{0}, K_{2}\right)\right.\right. \\
& \left.+b\left(F_{z}, K_{1}\right)\right]+w\left(F_{0}^{c}, F_{z}^{c}\right)+\left[a\left(F_{0}^{c}, K_{2}\right)+w\left(F_{0}^{c}, F_{z}\right)\right]\left[b\left(F_{z}^{c}, K_{1}\right)+v\left(F_{z}^{c}\right)+w\left(F_{0}, F_{z}^{c}\right)\right] \\
& \left.+u\left(F_{0}^{c}\right)\left[b\left(F_{z}^{c}, K_{1}\right)+w\left(F_{0}, F_{z}^{c}\right)\right]\right) H_{K_{1}}\left(\Gamma, d y_{1}\right) H_{K_{2}}\left(\Gamma, d y_{2}\right) Q\left(d K_{1}\right) Q\left(d K_{2}\right) d x_{2} d x_{1} .
\end{aligned}
$$

Therefore,

$$
\begin{aligned}
s_{2}^{(m)} & \leq \lambda^{2} \mathbf{E}\left|\left(F_{0}^{c} \oplus \check{E}_{0}\right) \cap \check{Z}_{0}\right| \mathbf{E} H_{Z_{0}}^{2}\left(\Gamma, \mathbb{R}^{d}\right) \\
& +\left(\lambda \mathbf{E}\left|\left(F_{0}^{c} \oplus \check{E}_{0}\right) \cap \check{Z}_{0}\right|\right)^{2}\left(\lambda \bar{H}\left(\Gamma, \mathbb{R}^{d}\right)^{2} \mathbf{E}\left|Z_{0}\right|^{2}+2 \bar{H}\left(\Gamma, \mathbb{R}^{d}\right) \mathbf{E}\left|Z_{0}\right| H_{Z_{0}}\left(\Gamma, \mathbb{R}^{d}\right)\right. \\
& +\mathbf{E}\left|\left(F_{0}^{c} \oplus \check{E}_{0}\right) \cap \check{Z}_{0}\right| H_{Z_{0}}\left(\Gamma, \mathbb{R}^{d}\right)\left(1+2 \lambda \mathbf{E}\left|Z_{0}\right|\right) \bar{H}\left(\Gamma, \mathbb{R}^{d}\right) \\
& +\mathbf{E}\left|\left(F_{0}^{c} \oplus \check{E}_{0}\right) \cap \check{Z}_{0}\right|\left|Z_{0}\right|\left(2 \lambda+3 \lambda^{2} \mathbf{E}\left|Z_{0}\right|\right)\left(\bar{H}\left(\Gamma, \mathbb{R}^{d}\right)\right)^{2},
\end{aligned}
$$

whence, arguing as above, it follows that $s_{2}^{(m)} \rightarrow 0$ as $m \rightarrow \infty$ which completes the proof of Theorem 7.1.

Corollary 7.2. If $\Psi$ is a stationary Poisson process with intensity $\lambda$, and $H$ admits integral representation (3.9) such that $\mathbf{E}\left|Z_{0}\right|^{2}<\infty$ and $\mathbf{E} \Upsilon_{Z_{0}}^{2}\left(\Gamma_{i}\right)<\infty$ for $i=1, \ldots, k$, then $\left(\hat{\eta}\left(\Gamma_{1}, W_{n}\right), \ldots, \hat{\eta}\left(\Gamma_{k}, W_{n}\right)\right)$ converges in distribution as $n \rightarrow \infty$ to a Gaussian centred random vector $\left(\xi_{1}, \ldots, \xi_{k}\right)$ having the covariance matrix

$$
\begin{aligned}
\mathbf{E} \xi_{i} \xi_{j}=\sigma^{2}\left(\Gamma_{i}, \Gamma_{j}\right) & =\lambda(1-p)^{2} \mathbf{E}\left[\iint_{\Gamma_{i}} q\left(\zeta_{\Gamma_{j}}, u_{2}\right) \Upsilon_{Z_{0}}\left(d u_{1}\right) \Upsilon_{Z_{0}}\left(d u_{2}\right)\right] \\
& +\lambda^{2}(1-p)^{2} \int_{\mathbb{R}^{d}}\left(q(x) \Phi\left(x, \Gamma_{i}\right) \Phi\left(-x, \Gamma_{j}\right)-\mathbf{E} \Upsilon_{Z_{0}}\left(\Gamma_{i}\right) \mathbf{E} \Upsilon_{Z_{0}}\left(\Gamma_{j}\right)\right) d x
\end{aligned}
$$

where

$$
\zeta_{u_{1}, u_{2}}=\ell\left(u_{1}, Z_{0}\right)-\ell\left(u_{2}, Z_{0}\right)
$$

and

$$
\Phi(x, \Gamma)=\mathbf{E}\left[\int_{\Gamma}\left(1-\mathbf{1}_{\check{Z}_{0}+\ell\left(u, Z_{0}\right)}(x)\right) \Upsilon_{Z_{0}}(d u)\right]
$$

In particular, if $\mathbb{U}=\left\{u_{1}, \ldots, u_{k}\right\}$ consists of $k$ distinct points, $\Gamma_{i}=\left\{u_{i}\right\}, 1 \leq i \leq k$, and $\Upsilon_{Z_{0}}=\delta_{u_{1}}+\cdots+\delta_{u_{k}}$ is a deterministic counting measure, then

$$
\sigma^{2}\left(\Gamma_{i}, \Gamma_{j}\right)=\lambda(1-p)^{2} \mathbf{E}\left[q\left(\zeta_{u_{1}, u_{2}}\right)\right]+\lambda^{2}(1-p)^{2} \int_{\mathbb{R}^{d}}\left(q(x) \phi_{u_{i}}(x) \phi_{u_{j}}(-x)-1\right) d x
$$

for $1 \leq i, j \leq k$, where

$$
\phi_{u}(x)=\mathbf{P}\left\{x \notin \check{Z}_{0}+\ell\left(u, Z_{0}\right)\right\}
$$




\section{Extensions of Geometric Functionals}

Different geometric functionals on the family of convex sets have been defined long ago, see, e.g., [6] and [21] for more recent developments. The most important are intrinsic volumes $V_{0}(K), \ldots, V_{d}(K)$ of a convex compact set $K[21, \mathrm{p} .210]$. These functionals can be defined from the coefficients of the polynomial expansion

$$
\left|K^{r}\right|=\sum_{j=0}^{d} b_{d-j} r^{d-j} V_{j}(K), \quad r \geq 0
$$

for the volume of the set $K^{r}=K \oplus B_{r}(0)$. Here $b_{d-j}$ denotes the volume of the unit ball in the space $\mathbb{R}^{d-j}$. Note that $V_{0}(K)=1$ for all convex $K, V_{d-1}(K)$ is one half of the surface area of $K$, and $V_{d}(K)=|K|$.

The intrinsic volumes are basically defined on the family of compact convex sets $\mathcal{C}$. Suppose now that $F$ belongs to the convex ring $\mathcal{R}$, i.e., $F$ is a finite union of convex compact sets $K_{i}, 1 \leq i \leq n$, i.e.,

$$
F=\bigcup_{i=1}^{n} K_{i}
$$

It is often important to extend geometric functionals onto the convex ring $\mathcal{R}$. There are two standard ways of doing this, see [21, 25]. The first is the additive extension given by

$$
V_{j}(F)=\sum_{k=1}^{n}(-1)^{k-1} \sum_{1 \leq i_{1}<\cdots<i_{k} \leq n} V_{j}\left(K_{i_{1}} \cap \cdots \cap K_{i_{k}}\right) .
$$

The resulting functionals on $\mathcal{R}$ are additive, but can be negative for $j \leq d-2$. Similarly to (8.1), it is possible to define these additive extensions via coefficients of the polynomial expansion of $\left|F^{r}\right|$.

In the following we will deal with the positive extension defined as follows (see [20, 21, 25 ] for further details). For given $F \in \mathcal{R}$ and $x \in \mathbb{R}^{d}$, a point $q \in F$ is called a projection of $x$ onto $F$ if there exists a neighbourhood $G$ of $q$ such that $q$ is the nearest point to $x$ among all points from $F \cap G$. Let $\Pi(F, x)$ be the set of all projections of $x$. For $\Gamma \subset \mathbb{S}^{d-1}$ and $r>0$ let

$$
\bar{c}_{r}(F, \Gamma \times W, x)=\#\{q \in \Pi(F, x): 0<\|x-q\| \leq r ; q \in W \text { and } \overline{x-q} \in \Gamma\}
$$

where $\overline{x-q}$ is the unit vector with the same direction as $x-q$. Furthermore, let

$$
\bar{\mu}_{r}(F, \Gamma \times W)=\int_{\mathbb{R}^{d}} \bar{c}_{r}(F, \Gamma \times W, x) d x .
$$

This measure can be decomposed in the following way:

$$
\bar{\mu}_{r}(F, \Gamma \times W)=\frac{1}{d} \sum_{j=0}^{d-1} r^{d-j}\left(\begin{array}{l}
d \\
j
\end{array}\right) \bar{\Theta}_{j}(F, \Gamma \times W)
$$


see [20]. If $K \in \mathcal{C}$, then we write $\Theta_{j}(K, \Gamma \times W)$ instead of $\bar{\Theta}_{j}(K, \Gamma \times W)$ and call it the $j$ th (generalized) curvature measure of $K$. For $F \in \mathcal{R}$, the coefficients $\bar{\Theta}_{j}(F, \Gamma \times W)$ in (8.4) are called the positive extension of the curvature measures. In particular,

$$
\bar{V}_{j}(F)=\frac{1}{d b_{d-j}}\left(\begin{array}{l}
d \\
j
\end{array}\right) \bar{\Theta}_{j}\left(F, \mathbb{S}^{d-1} \times \mathbb{R}^{d}\right), \quad F \in \mathcal{R},
$$

is the positive extension of the intrinsic volume $V_{j}$. Note that $\bar{V}_{d}(F)=V_{d}(F)$ and $\bar{V}_{d-1}(F)=V_{d-1}(F)$, so that the positive and additive extensions of orders $d$ and $d-1$ coincide. For instance, in the smooth case, $\bar{\Theta}_{d-1}(F, \Gamma \times W)$ can be interpreted as the $(d-1)$-dimensional Hausdorff measure (surface area) of all points in $(\partial F) \cap W$ such that the corresponding outer normals to $F$ lie in $\Gamma$.

Formula (3.7) suggests another way to define functionals on the convex ring. Let $H_{K}(\cdot)$ be a measure on $[\Sigma, \mathfrak{B}(\Sigma)]$, where $K \in \mathcal{C}$. Then

$$
\eta_{F}(\Gamma, W)=\sum_{i=1}^{n} H_{K_{i}}\left(\Gamma, W \backslash \cup_{1 \leq j \leq n, j \neq i} K_{j}\right) .
$$

gives a kind of extension of $H_{(\cdot)}(\Gamma, W)$ for any $F \in \mathcal{R}$ such that $F=\cup_{i=1}^{n} K_{i}$. Unfortunately, in general, this extension does depend on the decomposition of $F$ as the union of convex sets. Indeed, for any convex $F$ we can get $\eta_{F}(\Gamma, W)=0$ by using the trivial representation $F=F \cup F$. However, as it will be shown, such a situation is not possible if $F$ is a realization of a germ-grain models satisfying rather weak assumptions.

The most important example appears in the standard framework, where $\mathbb{U}=\mathbb{S}^{d-1}$ and $H_{K}(\Gamma, W)=\Theta_{j}(K, \Gamma \times W)$ is the $j$ th curvature measure of $K \in \mathcal{C}$. This is motivated by the possibility to get positive extensions of intrinsic volumes or curvature measures via integration of absolute curvatures over the boundaries of sets [1,29, 31], which is similar to the integral representation defined in (3.9).

If $F \in \mathcal{R}$, then $x \in \partial F$ is said to be an exposed positive tangent point if $x=\Pi(F, v)$ for some $v \notin F$. The set of all exposed positive tangent points of $F \in \mathcal{R}$ is denoted by $\partial^{+} F$ and is said to be the positive boundary of $F$. The set-difference $\partial F \backslash \partial^{+} F$ is comprised of sets of dimensions not greater than $(d-2)$. Note that $\partial^{+} F$ contains the set

$$
\mathcal{L}\left(K_{1}, \ldots, K_{n}\right)=\bigcup_{i=1}^{n}\left(\partial K_{i} \backslash \cup_{1 \leq j \leq n, j \neq i} K_{j}\right)
$$

for each decomposition $F=\cup_{i=1}^{n} K_{i}$ of $F$ into union of convex compact sets. Clearly, $\mathcal{L}(K, K)=\emptyset$.

Let us use (8.5) to extend onto $\mathcal{R}$ the measure $H_{K}(\Gamma, W)=\bar{\mu}_{r}(K, \Gamma \times W)$. For the moment, we suppose that $F=\cup_{i=1}^{n} K_{i}$ with

$$
\mathcal{L}\left(K_{1}, \ldots, K_{n}\right)=\partial^{+} F .
$$

This means that the positive boundary of $F$ is equal to the union of all "visible" (or exposed) boundaries of separate grains. Similar conditions appear in [30] when considering unions of sets of positive reach. 
By (8.3) and (8.6),

$$
\bar{\mu}_{r}(F, \Gamma \times W)=\sum_{i=1}^{n} H_{K_{i}}\left(\Gamma, W \backslash \cup_{1 \leq j \leq n, j \neq i} K_{j}\right) .
$$

Expanding both sides in the polynomials (8.4) shows immediately that the basic formula (8.5) applied to the curvature measure $H_{K}(\Gamma, W)=\Theta_{j}(K, \Gamma \times W), K \in \mathcal{C}$, gives its positive extension onto the convex ring, i.e., $\eta_{F}(\Gamma, W)=\bar{\Theta}_{j}(F, \Gamma \times W)$.

Let us consider an integral representation of the measure $H_{K}(\cdot)$. For convex set $K$ and direction $u\left(u \in \mathbb{U}=\mathbb{S}^{d-1}\right)$ define the support set

$$
\mathcal{L}(u, K)=\{x \in \partial K:\langle u, x\rangle=-h(K, u)\}
$$

where $\langle u, x\rangle$ is the scalar product and

$$
h(K, u)=\sup \{\langle u, x\rangle: x \in K\}
$$

is the support function of $K$. The point $\ell(u, K)$ is defined to be the lexicographical minimum of the set $\mathcal{L}(u, K)$ (if $\mathcal{L}(u, K)$ is a singleton, then $\mathcal{L}(u, K)=\{\ell(u, K)\}$ ). Then $\ell(u, K)$ is said to be the tangent point of $K$ in the direction $u$, see [18]. In fact, other choices of such a "specific" point $\ell(u, K)$ are possible.

EXAMPLE 8.1. We consider the simplest case when the convex set $K$ has only one tangent point in each direction. This means that the support set $\mathcal{L}(u, \cdot)$ is a singleton for all $u \in \mathbb{S}^{d-1}$. First, notice that $\bar{c}_{r}\left(K, \cdot \times \mathbb{R}^{d}, x\right), K \in \mathcal{C}$, is a measure on $\mathbb{S}^{d-1}$ concentrated at a finite number of directions (namely from $x$ to its possible projections). Thus,

$$
\bar{c}_{r}(K, \Gamma \times W, x)=\sum_{i=1}^{n} \int_{\Gamma} \mathbf{1}_{W}(\ell(u, K)) \bar{c}_{r}(K, d u \times W, x) .
$$

Integration with respect to $x$ and polynomial expansion of both sides give

$$
H_{K}(\Gamma, W)=\Theta_{j}(K, \Gamma \times W)=\int_{\Gamma} \mathbf{1}_{W}(\ell(u, K)) \Upsilon_{K}(d u)
$$

where $\Upsilon_{K}(\Gamma)=\Theta_{j}\left(K, \Gamma \times \mathbb{R}^{d}\right), \Gamma \in \mathfrak{U}$, is the $j$ th area measure of $K$, see [21, p. 203]. Thus, the curvature measure admits integral representation (3.9).

In the following we give conditions to hold the identity (8.6) in case of the germ-grain model (3.1). For this, define the set-theoretic limit

$$
\begin{aligned}
\mathcal{L}\left(X_{i}+Z_{i} ; i \geq 1\right) & =\lim _{n \rightarrow \infty} \mathcal{L}\left(X_{i}+Z_{i} ; i \geq 1,\left(X_{i}+Z_{i}\right) \cap B_{n}(0) \neq \emptyset\right) \\
& =\bigcup_{i: i \geq 1}\left(\partial\left(X_{i}+Z_{i}\right) \backslash \Xi_{i}\right) .
\end{aligned}
$$


Theorem 8.2. Let $\Psi=\sum_{i: i \geq 1} \delta_{X_{i}}$ be a stationary second-order point process satisfying $\alpha_{\text {red }}^{(2)}(\cdot) \ll|\cdot|$, i.e., its second-order reduced moment measure is absolutely continuous with respect to the Lebesgue measure in $\mathbb{R}^{d}$. Further assume that the typical grain $Z_{0}$ is a.s. compact and convex. Then, for $Z$ defined in (3.1), we have

$$
\mathbf{P}\left\{\mathcal{L}\left(X_{i}+Z_{i} ; i \geq 1\right)=\partial^{+} Z\right\}=1
$$

Remark 6. Clearly, the conditions of Theorem 8.2 are valid for each Boolean model with any almost surely convex compact typical grain.

Proof. Let $K_{1}$ and $K_{2}$ be two convex compact sets. Then $\mathcal{L}\left(K_{1}, K_{2}\right) \neq \partial^{+}\left(K_{1} \cup K_{2}\right)$ implies that either $\mathcal{L}\left(u, K_{1}\right) \cap \mathcal{L}\left(u, K_{2}\right) \neq \emptyset$ or $\mathcal{L}\left(u, K_{1}\right) \cap \mathcal{L}\left(-u, K_{2}\right) \neq \emptyset$ for some $u \in \mathbb{S}^{d-1}$. Thus,

$$
\begin{aligned}
& L\left(K_{1}, K_{2}\right)=\left\{(x, y): \mathcal{L}\left(K_{1}+x, K_{2}+y\right) \neq \partial^{+}\left(\left(K_{1}+x\right) \cup\left(K_{2}+y\right)\right)\right\} \\
& \quad \subseteq \bigcup_{u \in \mathbb{S}^{d-1}}\left\{(x, y): y-x \in\left(\mathcal{L}\left(u, K_{1}\right) \oplus \check{\mathcal{L}}\left(u, K_{2}\right)\right) \cup\left(\mathcal{L}\left(u, K_{1}\right) \oplus \check{\mathcal{L}}\left(-u, K_{2}\right)\right)\right\} .
\end{aligned}
$$

It follows from Theorem 1.7 .5 of [21] that

$$
\mathcal{L}\left(u, K_{1}\right) \oplus \check{\mathcal{L}}\left(-u, K_{2}\right)=\mathcal{L}\left(u, K_{1}\right) \oplus \mathcal{L}\left(u, \check{K}_{2}\right)=\mathcal{L}\left(u, K_{1} \oplus \check{K}_{2}\right)
$$

Thus,

$$
L\left(K_{1}, K_{2}\right) \subseteq\left\{(x, y): y-x \in \partial\left(K_{1} \oplus \check{K}_{2}\right) \cup \Lambda\left(K_{1}, K_{2}\right)\right\}
$$

where

$$
\Lambda\left(K_{1}, K_{2}\right)=\bigcup_{u \in \mathbb{S}^{d-1}}\left[\mathcal{L}\left(u, K_{1}\right) \oplus \check{\mathcal{L}}\left(u, K_{2}\right)\right]
$$

see $[21$, p. 86]. The technique described in [21, Section 2.3] can be used to prove that the Lebesgue measure of $\Lambda\left(K_{1}, K_{2}\right)$ is equal to zero. First, the equality $\mathcal{L}\left(u, K_{1} \oplus B_{1}(0)\right)=$ $\mathcal{L}\left(u, K_{1}\right)+u$ allows to consider sets $K_{1}$ and $K_{2}$ which contain a ball of radius 1. Therefore, Lemma 2.3.9 of [21] yields

$$
\Lambda\left(K_{1}, K_{2}\right) \subset \bigcup_{i=1}^{m}\left(\left(C_{i}+\check{C}_{i}\right)+a_{i}\right),
$$

where $a_{i} \in \mathbb{R}^{d}$ and $C_{1}, \ldots, C_{m}$ are caps of $K_{1} \oplus K_{2}$ covering the boundary of $K_{1} \oplus K_{2}$. A cap is defined to be a non-empty intersection of the convex set with a closed half-space. Furthermore, Theorem 2.3.2 [21] gives a possibility to choose these caps in such a way that

$$
\sum_{i=1}^{m}\left|C_{i}\right|<\varepsilon
$$

for any given $\varepsilon>0$. Note that $\left|C_{i} \oplus \check{C}_{i}\right| \leq(d+1)^{d}\left|C_{i}\right|$. The latter follows from the fact that $\breve{C} \subset d C$ for any convex compact set $C$ with non-empty interior and having its centroid at the origin [21, p. 81]. Thus, the Lebesgue measure of the set in the right-hand side of (8.8) can be made arbitrarily small. Thus, $L\left(K_{1}, K_{2}\right) \subseteq\left\{(x, y): y-x \in \tilde{L}\left(K_{1}, K_{2}\right)\right\}$ with $\left|\tilde{L}\left(K_{1}, K_{2}\right)\right|=0$. 
Now consider the germ-grain model $Z$ defined by (3.1). Notice that

$$
\begin{aligned}
\left\{\mathcal { L } \left(Z_{1}+X_{1},\right.\right. & \left.\left.Z_{2}+X_{2}, \ldots\right) \neq \partial^{+} Z\right\} \\
& \subseteq \bigcup_{i \neq j}\left\{\mathcal{L}\left(Z_{i}+X_{i}, Z_{j}+X_{j}\right) \neq \partial^{+}\left(\left(Z_{i}+X_{i}\right) \cup\left(Z_{j}+X_{j}\right)\right)\right\} \\
& =\bigcup_{i \neq j}\left\{\left(X_{i}, X_{j}\right) \in L\left(Z_{i}, Z_{j}\right)\right\} \\
& =\bigcup_{i \neq j}\left\{\left(X_{i}-X_{j}\right) \in \tilde{L}\left(Z_{i}, Z_{j}\right)\right\} .
\end{aligned}
$$

The $\mathbf{P}$-measure of the right-hand side of the last line equals the limit (as $n \rightarrow \infty$ ) of the probabilities

$$
\begin{aligned}
& \mathbf{P}\left\{\bigcup_{i \neq j: X_{i}, X_{j} \in B_{n}(0)}\left\{X_{i}-X_{j} \in \tilde{L}\left(Z_{i}, Z_{j}\right)\right\}\right\} \\
& \leq \int_{B_{n}(0) \times B_{n}(0)} \int_{\mathcal{K} \times \mathcal{K}} \mathbf{1}_{\tilde{L}\left(L_{1}, L_{2}\right)}(x-y) Q\left(d L_{1}\right) Q\left(d L_{2}\right) \alpha^{(2)}(d(x, y)) \\
& \quad=\lambda \int_{\mathcal{C} \times \mathcal{C}} \int_{B_{n}(0)} \alpha_{\text {red }}^{(2)}\left(\tilde{L}\left(L_{1}, L_{2}\right) \cap B_{n}(y)\right) d y Q\left(d L_{1}\right) Q\left(d L_{2}\right) .
\end{aligned}
$$

Since $\left|\tilde{L}\left(L_{1}, L_{2}\right)\right|=0$ for any $L_{1}, L_{2} \in \mathcal{C}$ and by the assumptions of Theorem 8.2 the latter integral is equal to zero for every $n \geq 1$. This completes the proof of Theorem 8.2.

Theorem 8.2 implies that, for $H_{K}(\Gamma, W)=\Theta_{j}(K, \Gamma \times W), K \in \mathcal{C}$, the corresponding measure $\eta$ associated with $Z$ coincides almost surely with the positive extension of the $j$ th generalized curvature measure of $Z$.

\section{Statistical Applications and Examples}

In this section we consider only Boolean models. In this case the Poisson germ process is determined by only one parameter (the intensity $\lambda$ ), while for the typical grain the mean values of geometric functionals are usually estimated.

The most severe difficulty in statistics of the Boolean model is caused by overlappings of the grains. One of standard estimation techniques for the Boolean model uses the following idea $[18,22,26]$. Take a geometric functional of the typical grain (if the grain is convex, then one of the intrinsic volumes is a possible choice). Then extend it onto the convex ring and compute its spatial intensity. This empirical spatial intensity is then plugged into an equation related its theoretical counterpart to $\lambda$ and the parameters of the typical grain. For example, the volume fraction is equal to $p=1-\exp \left\{-\lambda \mathbf{E}\left|Z_{0}\right|\right\}$, which allows to estimate $\lambda \mathbf{E}\left|Z_{0}\right|$ after replacing $p$ by its empirical counterpart.

This procedure is known as the methods of moments in statistics of the Boolean model, see [17]. The details depend on the chosen functionals and the way of their extension used 
in this procedure. We will use the positive extension, which is closely related to random measures given by (3.7).

According to what has been said, the statistical estimation begins with the choice of the measure $H_{K}(\cdot)$ on $[\Sigma, \mathfrak{B}(\Sigma)]$. This measure is used to define the random measure $\eta$ associated with the underlying Boolean model. Then Lemma 4.1 and Theorem 6.1 yield

$$
\frac{\eta(\Gamma, W)}{|W|\left(1-\hat{p}_{W}\right)} \rightarrow \lambda \bar{H}\left(\Gamma, \mathbb{R}^{d}\right) \quad \text { as } \quad W \uparrow \mathbb{R}^{d}
$$

where $\hat{p}_{W}=|W \cap Z| /|W|$ estimates the volume fraction of $Z$. For the estimation of $\lambda$ we put $\Gamma=\mathbb{U}$ and take any measure $H_{K}(\cdot)$ with known $\bar{H}\left(\mathbb{U}, \mathbb{R}^{d}\right)=\mathbf{E} H_{Z_{0}}\left(\mathbb{U}, \mathbb{R}^{d}\right)$ (for example, any probability measure on $\Sigma$ will do). Then the estimator of $\lambda$ can be plugged again into (9.1) to estimate $\bar{H}\left(\Gamma, \mathbb{R}^{d}\right)$ for another $H_{K}(\cdot)$.

Below we will prove a central limit theorem for the random measure

$$
\tilde{\eta}\left(\Gamma, W_{n}\right)=\left|W_{n}\right|^{1 / 2}\left(\frac{\eta\left(\Gamma, W_{n}\right)}{\left|W_{n}\right|\left(1-\hat{p}_{W_{n}}\right)}-\lambda \bar{H}\left(\Gamma, \mathbb{R}^{d}\right)\right),
$$

where $W_{n}=[-n, n)^{d}$ and $n \rightarrow \infty$.

Theorem 9.1. Under conditions of Theorem 7.1 , the random vector $\tilde{\eta}\left(\Gamma_{1}, W_{n}\right), \ldots$, $\tilde{\eta}\left(\Gamma_{k}, W_{n}\right)$ converges in distribution to a centred Gaussian random vector with the covariances

$$
\sigma_{\Gamma_{i} \Gamma_{j}}^{2}=\lambda \int_{\left(\mathbb{R}^{d}\right)^{2}} q\left(y_{1}-y_{2}\right) \bar{H}\left(\Gamma_{i}, d y_{1} ; \Gamma_{j}, d y_{2}\right)+\lambda^{2} \int_{\mathbb{R}^{d}} \tilde{\Phi}\left(x, \Gamma_{i}\right) \tilde{\Phi}\left(-x, \Gamma_{j}\right) q(x) d x
$$

where $i, j=1, \ldots, k$, and

$$
\left.\tilde{\Phi}(x, \Gamma)=\mathbf{E}\left[H_{Z_{0}}\left(\Gamma, Z_{0}+x\right)\right)\right]
$$

i.e., $\tilde{\Phi}(x, \Gamma)=\tilde{\Phi}\left(x, \Gamma, \mathbb{R}^{d}\right)$, see $(6.10)$.

Proof. Similarly to [18, Theorem 5.5], it suffices to calculate the mixed moment of $\eta\left(\Gamma, W_{n}\right)$ and $\hat{p}_{n}=\hat{p}_{W_{n}}$. First, note that

$$
1-\hat{p}_{n}=\left|W_{n}\right|^{-1} \int_{W_{n}}\left[1-\mathbf{1}_{Z}(x)\right] d x=\left|W_{n}\right|^{-1} \int_{W_{n}} \prod_{j: j \geq 1}\left(1-\mathbf{1}_{X_{j}+Z_{j}}(x)\right) d x .
$$

Now we deduce

$$
\begin{aligned}
\mathbf{E}\left[\eta\left(\Gamma, W_{n}\right)\left(1-\hat{p}_{n}\right)\right]=\left|W_{n}\right|^{-1} \int_{W_{n}} \mathbf{E}\left[\sum_{i: i \geq 1} \int_{\mathbb{R}^{d}} \mathbf{1}_{W_{n}}\left(X_{i}+y\right)\left(1-\mathbf{1}_{X_{i}+Z_{i}}(x)\right)\right. \\
\left.\times \prod_{j: j \neq i}\left\{\left(1-\mathbf{1}_{X_{j}+Z_{j}}(x)\right)\left(1-\mathbf{1}_{X_{j}+Z_{j}}\left(X_{i}+y\right)\right)\right\} H_{Z_{i}}(\Gamma, d y)\right] d x .
\end{aligned}
$$


The refined Campbell theorem yields

$$
\begin{gathered}
\mathbf{E}\left[\eta\left(\Gamma, W_{n}\right)\left(1-\hat{p}_{n}\right)\right]=\lambda\left|W_{n}\right|^{-1} \int_{W_{n}} \int_{\mathbb{R}^{d}} \int_{\mathcal{K}} \int_{\mathbb{R}^{d}} \mathbf{1}_{W_{n}}(z+y)\left(1-\mathbf{1}_{\check{K}}(z-x)\right) \\
\quad \times G_{0}^{!}\left[\mathbf{P}\left\{(\cdot) \notin\left(\check{Z}_{0}+x-z\right) \cup\left(\check{Z}_{0}+y\right)\right\}\right] H_{K}(\Gamma, d y) Q(d K) d z d x .
\end{gathered}
$$

In particular, we have used the identity

$$
\left.\left.\left(1-\mathbf{1}_{X_{j}+Z_{j}}(x)\right)\left(1-\mathbf{1}_{Z_{j}+Z_{j}}\left(X_{i}+y\right)\right)=\mathbf{1}_{\check{Z}_{j}^{c}}\left(X_{j}-X_{i}+X_{i}-x\right)\right) \mathbf{1}_{\check{Z}_{j}^{c}}\left(X_{j}-X_{i}-y\right)\right) .
$$

Substituting $z+y=v+x$ yields

$$
\begin{aligned}
\mathbf{E}[\eta( & \left.\left.\Gamma, W_{n}\right)\left(1-\hat{p}_{n}\right)\right]=\lambda\left|W_{n}\right|^{-1} \iint_{W_{n}} \int_{\mathbb{R}^{d}} \int_{\mathbb{K}^{d}} \mathbf{1}_{W_{n}}(v+x) \mathbf{1}_{\check{K}^{c}}(v-y) \\
& \times G_{0}^{!}\left[\mathbf{P}\left\{(\cdot) \notin\left(\check{Z}_{0}+y-v\right) \cup\left(\check{Z}_{0}+y\right)\right\}\right] H_{K}(\Gamma, d u) Q(d K) d v d x \\
= & \lambda \int_{\mathbb{R}^{d}} \gamma_{W_{n}}(v)\left|W_{n}\right|^{-1} \int_{\mathcal{K}_{\mathbb{R}^{d}}} \mathbf{1}_{\check{K}^{c}}(v-y) \\
& \times G_{0}^{!}\left[\mathbf{P}\left\{(\cdot) \notin\left(\check{Z}_{0}+y-v\right) \cup\left(\check{Z}_{0}+y\right)\right\}\right] H_{K}(\Gamma, d y) Q(d K) d v .
\end{aligned}
$$

The calculations above were made without referring to the Poisson assumption. Now we assume that the point process $\Psi$ is Poisson. Then the value of the probability generating functional in the latter integral does not depend on $y$ and is given by

$$
\begin{aligned}
G_{0}^{!}[\mathbf{P}\{(\cdot) & \left.\left.\notin\left(\check{Z}_{0}+y-v\right) \cup\left(\check{Z}_{0}+y\right)\right\}\right] \\
& =\exp \left\{-\lambda \int_{\mathbb{R}^{d}}\left[1-\left(1-\mathbf{E} \mathbf{1}_{\check{Z}_{0}}(u+v)\right)\left(1-\mathbf{E} \mathbf{1}_{\check{Z}_{0}}(u)\right)\right] d u\right\} \\
& =\exp \left\{-\lambda\left(2 \mathbf{E}\left|Z_{0}\right|-\mathbf{E}\left|Z_{0} \cap\left(Z_{0}+v\right)\right|\right)\right\} \\
& =(1-p)^{2} q(v) .
\end{aligned}
$$

Finally,

$$
\mathbf{E}\left[\eta\left(\Gamma, W_{n}\right)\left(1-\hat{p}_{n}\right)\right]=\lambda(1-p)^{2} \int_{\mathbb{R}^{d}} \gamma_{W_{n}}(v)\left|W_{n}\right|^{-1} \int_{\mathbb{R}^{d}}\left(1-\mathbf{1}_{\check{Z}_{0}+y}(v)\right) \bar{H}(\Gamma, d y) q(v) d v .
$$

The proof can be easily accomplished by elementary calculations.

Corollary 9.2. Suppose that $H$ admits integral representation (3.9). Then, under conditions of Theorem 7.1, the statement of Theorem 9.1 holds with the covariances given by

$$
\sigma_{\Gamma_{i} \Gamma_{j}}^{2}=\lambda \mathbf{E}\left[\int_{\Gamma_{i}} \int_{\Gamma_{j}} q\left(\zeta_{u_{1}, u_{2}}\right) \Upsilon_{Z_{0}}\left(d u_{1}\right) \Upsilon_{Z_{0}}\left(d u_{2}\right)\right]+\lambda^{2} \int_{\mathbb{R}^{d}} \tilde{\Phi}\left(x, \Gamma_{i}\right) \tilde{\Phi}\left(-x, \Gamma_{j}\right) q(x) d x
$$


where $\zeta_{u_{1}, u_{2}}$ is defined in (7.6), and

$$
\tilde{\Phi}(x, \Gamma)=\mathbf{E}\left[\int_{\Gamma} \mathbf{1}_{Z_{0}+\ell\left(u, Z_{0}\right)}(x) \Upsilon_{Z_{0}}(d u)\right] .
$$

In particular, if $\mathbb{U}=\left\{u_{1}, \ldots, u_{n}\right\}$, and $\Upsilon_{Z_{0}}(\cdot)$ is the deterministic counting measure, then

$$
\sigma_{u_{i} u_{j}}^{2}=\lambda \mathbf{E} q\left(\zeta_{u_{1}, u_{2}}\right)+\lambda^{2} \int_{\mathbb{R}^{d}} \tilde{\phi}_{u_{i}}(x) \tilde{\phi}_{u_{j}}(-x) q(x) d x
$$

where

$$
\tilde{\phi}_{u}(x)=1-\phi_{u}(x)=\mathbf{P}\left\{x \in \check{Z}_{0}+\ell\left(u, Z_{0}\right)\right\}
$$

Now we consider several examples of measures $H_{K}(\cdot)$ and statistical applications of the above asymptotic theory. Note that $\eta$ is defined by formula (3.7), which includes individual grains from the underlying germ-grain model. However, only observations of the union-set $Z$ are available for the statistical analysis.

ExAmple 9.3. Let $H_{K}(\Gamma, W)=|K \cap W|$ being independent of $\Gamma$, i.e., $\mathbb{U}$ is trivial (consists of one point). Then $\eta(\Gamma, W)$ is equal to the Lebesgue measure of all points covered by $Z_{i}+X_{i}$ for only one $i$. Clearly, the values of the measure $\eta$ cannot be computed if only the union-set $Z$ is observable. If $\mathbf{E} H_{Z_{0}}\left(\Gamma, \mathbb{R}^{d}\right)=\mathbf{E}\left|Z_{0}\right|<\infty$, then Theorem 6.1 yields

$$
|W|^{-1} \eta(\Gamma, W) \rightarrow \lambda(1-p) \mathbf{E}\left|Z_{0}\right| \quad \text { a.s. as } W \uparrow \mathbb{R}^{d}
$$

Furthermore, if $\mathbf{E}\left|Z_{0}\right|^{2}<\infty$, then $\hat{\eta}\left(\Gamma, W_{n}\right)$ given by (6.3) satisfies the central limit theorem (Theorem 7.1) with the limiting variance given by

$$
\begin{aligned}
\sigma^{2}= & \lambda(1-p)^{2} \mathbf{E}\left[\int_{Z_{0}} \int_{Z_{0}} q\left(y_{1}-y_{2}\right) d y_{1} d y_{2}\right] \\
& +\lambda^{2}(1-p)^{2} \int_{\mathbb{R}^{d}}\left[q(x) \mathbf{E}\left|Z_{0} \cap\left(Z_{0}^{c}+x\right)\right| \mathbf{E}\left|Z_{0} \cap\left(Z_{0}^{c}-x\right)\right|-\left(\mathbf{E}\left|Z_{0}\right|\right)^{2}\right] d x .
\end{aligned}
$$

In statistical applications it is necessary to be able to observe $\eta(\Gamma, W)$ in order to make further calculations with the corresponding estimators. Most of the interesting examples appear in the case when $\mathbb{U}$ is the unit sphere $\mathbb{S}^{d-1}$ in $\mathbb{R}^{d}$, and $H$ admits an integral representation such that the points $\ell\left(u, Z_{i}\right) \backslash \Xi_{i}$ are observable. Unless otherwise stated the typical grain $Z_{0}$ is supposed to be almost surely compact and convex. Then $\ell\left(u, Z_{i}\right)$ can be the tangent point of $Z_{i}$ in the direction $u \in \mathbb{S}^{d-1}$. Fortunately, the tangent points are observable inside the interior of the window $W$ and these points may occur on the boundary of $W$ only with probability zero.

EXAMPLE 9.4. Let $H_{K}(\cdot)$ admit integral representation (3.9) with $\Upsilon_{Z_{0}}$ concentrated at a single point $u \in \mathbb{U}$ such that $\Upsilon_{Z_{0}}(\{u\})=1$. Then $H_{K}(\cdot)$ is a probability measure on $\Sigma$ given by

$$
H_{K}(\Gamma, W)=\mathbf{1}_{W}(\ell(u, K)), \quad u \in \Gamma .
$$


Therefore, the $i$ th term in (3.7) is 1 if and only if $\ell\left(u, Z_{i}+X_{i}\right) \in W \backslash \Xi_{i}$. The latter means that the specific point, $\ell\left(u, Z_{i}+X_{i}\right)$, of the $i$ th grain is exposed, i.e., not covered by the grains $\left(Z_{j}+X_{j}\right)$ with $j \neq i$. Then, for all $\Gamma \ni u, \eta(\Gamma, W)$ is equal to the number of exposed specific points lying in $W$, i.e.,

$$
\eta(\Gamma, W)=\#\left\{i: \ell\left(u, Z_{i}\right)+x_{i} \in W \backslash \Xi_{i}\right\} .
$$

For example, if $\ell(u, K)$ is the tangent point of $K$ in the direction $u$, then $\eta(\Gamma, W)$ is exactly the number $N^{+}(u, W)$ of exposed tangent points in the direction $u$, see [18, 17]. Since $\eta(\Gamma, W)=N^{+}(u, W)$ is observable, and, by the ergodic theorem,

$$
\frac{\eta(\Gamma, W)}{|W|\left(1-\hat{p}_{W}\right)} \rightarrow \lambda \quad \text { a.s. as } \quad W \uparrow \mathbb{R}^{d}
$$

Therefore, it is possible to estimate the intensity of the Boolean model using the spatial intensity of $\eta$ (or the intensity of the point process of exposed tangent points). Furthermore, Lemma 4.2 gives moment measures for the point process of tangent points, and Corollary 9.2 yields a central limit theorem for the empirical estimator of the intensity of this point process. In this case (9.7) implies

$$
\sigma_{u u}^{2}=\frac{\lambda}{1-p}
$$

since $\tilde{\phi}_{u}(x) \tilde{\phi}_{u}(-x)=0$ for almost all $x$. Note that (9.9) gives the asymptotic variance of the intensity estimator based on counting of tangent points. This variance was computed directly in [18].

If $\Upsilon_{Z_{0}}=\Upsilon_{0}$ is a deterministic probability measure on $\mathbb{S}^{d-1}$, then

$$
\eta(\Gamma, W)=\int_{\Gamma} N^{+}(u, W) \Upsilon_{0}(d u) .
$$

This is exactly the weighted number of exposed tangent points considered in [17]. Then Corollary 9.2 yields Theorem 3.1 of [17], which gives the asymptotic variance of the corresponding estimator.

ExAmple 9.5. Suppose that the support set $\mathcal{L}\left(u, Z_{0}\right)=\left\{\ell\left(u, Z_{0}\right)\right\}$ is a singleton for all $u \in \mathbb{S}^{d-1}=\mathbb{U}$ and almost all realizations of $Z_{0}$. Let $k_{Z_{0}}(u)$ be a positive function which depends on $\partial Z_{0} \cap B_{\varepsilon}\left(\ell\left(u, Z_{0}\right)\right)$ for arbitrarily small $\varepsilon>0$. In particular, $k_{Z_{0}}(u)$ can be the absolute curvature of $\partial Z_{0}$ at the corresponding tangent point or a function of this curvature. The measure $H$ is supposed to admit integral representation (3.9) with $\Upsilon_{Z_{0}}(\cdot)$ concentrated at $\{u\}$ with mass 1 . Then

$$
\mathbf{E} \eta(\{u\}, W)=\lambda|W|(1-p) \mathbf{E} k_{Z_{0}}(u)
$$

whence the expected value of $k_{Z_{0}}(u)$ can be estimated as soon as an estimator of $\lambda$ is available. If $\mathbf{E}\left|Z_{0}\right|^{2}<\infty$ and $\mathbf{E} k_{Z_{0}}^{2}(u)<\infty$ for all $u$, then Corollary 9.2 is applicable with the limiting variance equal to

$$
\sigma_{u u}^{2}(k)=\lambda \mathbf{E}\left(k_{Z_{0}}(u)^{2}\right) /(1-p) .
$$


To estimate the expected value of $k_{Z_{0}}(u)$ one should use an estimator of $\lambda$ (e.g., based on counting of tangent points). For instance, if the grain is a random ball of radius $\xi$, then all moments of $\xi$ (and also all expectations $\mathbf{E} f(\xi)$ ) can be estimated.

EXAmple 9.6. Let $d=2, \mathbb{U}$ be the unit circle, and let $H_{K}(\Gamma, \cdot)$ with $\Gamma \ni u$ be concentrated at the tangent point $\ell(u, K)$ with mass 1 if the support set $\mathcal{L}(u, K)$ is a singleton. Otherwise, $\mathcal{L}(u, K)$ is a segment and $H_{K}(\Gamma, \cdot)$ gives the weights $1 / 2$ to its end-points denoted by $\ell^{\prime}(u, K)$ and $\ell^{\prime \prime}(u, K)$. Note that $H_{K}(\cdot)$ does not admit integral representation (3.9).

The corresponding measure $\eta$ is still observable and can be used to estimate the intensity of the Boolean model, since, by Theorem $6.1, \eta(\Gamma, W) /|W| \rightarrow \lambda(1-p)$ almost surely as $W \uparrow \mathbb{R}^{d}$. Theorem 9.1 implies that the asymptotic variance of the corresponding estimator, $\hat{\lambda}=\eta(\Gamma, W) /\left(|W|\left(1-\hat{p}_{W}\right)\right)$, is equal to

$$
\sigma_{u u}^{2}=\lambda \mathbf{E}\left[q(0)+q\left(\ell^{\prime \prime}\left(u, Z_{0}\right)-\ell^{\prime}\left(u, Z_{0}\right)\right)\right] / 2 .
$$

This variance is less than the variance given by (9.9) of the usual tangent points estimator, since $q(v) \leq q(0)=1 /(1-p), v \in \mathbb{R}^{d}$. Clearly, it is possible to consider other weights of the end-points or to write a higher-dimensional variant of this example.

ExAmple 9.7. Suppose that $\mathbb{U}=\mathbb{S}^{d-1}$, and $H_{Z_{0}}(\Gamma, W)=\Theta_{d-1}\left(Z_{0}, \Gamma \times W\right)$ is the $(d-1)$ dimensional generalized surface area measure of the typical grain. If $W$ is an open set, then $\eta(\Gamma, W)$ is equal to the surface area measure of the boundary of the germ-grain model $Z$ measured inside $W$. In particular, $\eta\left(\mathbb{S}^{d-1}, W\right)$ is equal to the surface area of $(\partial Z) \cap W$. Then the results above give the ergodic theorem and the central limit theorem for surface measures. In particular, the ergodic theorem yields an estimator of the mean surface area measure of the typical grain, which is equal to $\bar{H}\left(\Gamma, \mathbb{R}^{d}\right)$.

If the typical grain has no flat pieces on its boundary, then $H_{K}(\cdot)$ admits integral representation (3.9) with $\ell(u, K)$ being the tangent point and $\Upsilon_{Z_{0}}(\Gamma)=\Theta_{d-1}\left(Z_{0}, \Gamma \times\right.$ $\mathbb{R}^{d}$ ) being the area measure of order $(d-1)$, see [21, p. 203] and Example 8.1. Then Theorem 7.1 yields the limit theorem for surface measures proved in [17]. Note that it is possible to extend this example for non-convex grains with rectifiable boundaries.

If the window $W$ is closed, then the situation is more complicated, since the parts of the boundary of $W$ covered by $Z$ contribute to $\eta$, see [17]. In particular, it was shown in [17] that the central limit theorem is not valid in this case.

The following example was inspired by Hall [7, Section 5.6], who considered the planar Boolean model with a circular grain and statistics of the type

$$
\kappa(W)=\sum_{i: i \geq 1} \phi_{i} a\left(R_{i}\right)
$$

where $\phi_{i}$ is the angular content of the exposed boundary of the $i$ th grain (disk) within the window $W$, and $R_{i}$ is the radius of the $i$ th disk. In other words, $\kappa$ is the weighted sum of all angular contents of all protruded pieces of the boundary. In particular, Hall [7] proved that

$$
\mathbf{E} \kappa=2 \pi \lambda|W|(1-p) \mathbf{E} a(\xi),
$$

if $\mathbf{E} \xi^{2}<\infty$ and $\mathbf{E}|a(\xi)|<\infty$, where $\xi$ is the radius of the typical grain (disk). 
ExAmple 9.8. Let $H_{K}(\Gamma, W)=\Theta_{j}(K, \Gamma \times W) f(K)$, where $f(K)$ is a translation-invariant positive functional on the space of convex compact sets. The corresponding measure $\eta_{f}(\cdot)$ is observable as soon as the value $f(K)$ is retrievable from any relatively open piece of the boundary $\partial K$. In particular, this is true if the typical grain is a ball. Then statistics (9.10) can be obtained from $\eta_{f}(\cdot)$ for $d=2, \Gamma=\mathbb{U}=\mathbb{S}^{d-1}, j=1$, and $f\left(B_{\xi}(x)\right)=\xi^{-1} a(\xi)$. It follows from Lemma 4.1 that

$$
\mathbf{E} \eta_{f}(\Gamma, W)=\lambda|W|(1-p) \mathbf{E}\left[\Theta_{j}\left(Z_{0}, \Gamma \times \mathbb{R}^{d}\right) f\left(Z_{0}\right)\right],
$$

which yields (9.11) as a special case (for positive $f$ and circular grains). It is possible to use this approach to estimate $\mathbf{E} f\left(Z_{0}\right)$, since

$$
\mathbf{E} \eta_{f_{1}}(\Gamma, W)=\lambda|W|(1-p) \mathbf{E}\left[f\left(Z_{0}\right)\right]
$$

for $f_{1}=f(K) / \Theta_{j}\left(K, \Gamma \times \mathbb{R}^{d}\right)$.

If $\mathbf{E}\left|Z_{0}\right|^{2}<\infty$ and $\mathbf{E}\left[V_{j}\left(Z_{0}\right) f\left(Z_{0}\right)\right]^{2}<\infty$, then Theorem 7.1 implies that the finitedimensional distributions of $\tilde{\eta}\left(\Gamma, W_{n}\right)$ are asymptotically Gaussian with the covariance (7.2). These moment assumptions coincide with those imposed in [7, Theorem 5.3] for planar Boolean models with circular grains. In this case $H_{Z_{0}}(\cdot)$ admits integral representation (3.9) with $\Upsilon_{Z_{0}}(d u)=a(\xi) d u$ and $\ell\left(u, Z_{0}\right)$ equal to the tangent point of $Z_{0}$ in the direction $u$. Then Corollary 7.2 yields the asymptotic variance of $\kappa$

$$
\begin{aligned}
\sigma_{\kappa}^{2}= & \lambda(1-p)^{2} \mathbf{E}\left[a(\xi)^{2} \xi^{-2} \int_{\partial Z_{0}} \int_{\partial Z_{0}} q\left(y_{1}-y_{2}\right) d y_{1} d y_{2}\right] \\
& +\lambda^{2}(1-p)^{2} \int_{\mathbb{R}^{d}}\left(q(x) b(x) b(-x)-4 \pi^{2}(\mathbf{E} a(\xi))^{2}\right) d x,
\end{aligned}
$$

where the integrations over curves are understood with respect to the 1-dimensional Hausdorff measure (curve length), and

$$
b(x)=\mathbf{E}\left[a(\xi) \xi^{-1} \int_{\partial Z_{0} \cap\left(Z_{0}^{c}+x\right)} d y\right]
$$

is the expected angular content of $\partial Z_{0}$ within $Z_{0}^{c}+x$ multiplied by $a(\xi)$.

EXAMPLE 9.9. Let us consider typical grain $Z_{0}$ which has almost surely a bounded number of flat pieces on its boundary. Then for almost all realizations of $Z_{0}, \mathcal{H}^{(d-1)}\left(\mathcal{L}\left(u, Z_{0}\right)\right)>$ 0 for some finite set of points $u \in \mathbb{S}^{d-1}=\mathbb{U}$, where $\mathcal{H}^{(d-1)}(\cdot)$ is the $(d-1)$-dimensional Hausdorff measure. Put

$$
H_{Z_{0}}(\Gamma, W)=\sum_{u \in \Gamma, \mathcal{H}^{(d-1)}\left(\mathcal{L}\left(u, Z_{0}\right)\right)>0} \mathcal{H}^{(d-1)}\left(\mathcal{L}\left(u, Z_{0}\right) \cap W\right) .
$$

If $\mathbf{E}\left(\mathcal{H}^{(d-1)}\left(\partial Z_{0}\right)\right)^{2}<\infty$, then the corresponding measure $\eta$ is both ergodic and satisfies the central limit theorem. In particular, $|W|^{-1} \eta(\mathbb{U}, W) \rightarrow \lambda(1-p) S_{d-1}$, where $S_{d-1}$ is the expected value of the $(d-1)$-dimensional Hausdorff measure of all flat pieces in $\partial Z_{0}$. 
Furthermore,

$$
H_{Z_{0}}(\Gamma, W)=\#\left\{u \in \Gamma: \mathcal{H}^{(d-1)}\left(\mathcal{L}\left(u, Z_{0}\right) \cap W\right)>0\right\}
$$

is equal to the number of flat faces of $Z_{0}$ with normals belonging to $\Gamma$. Then $\eta(\mathbb{U}, W)$ is the number of exposed flat pieces in $W$. This gives a way to estimate the expected number of flat faces of the typical grain and, moreover, to formulate the central limit theorem for the corresponding estimator.

EXAMPle 9.10. Let $Z_{0}$ be a random convex polytope in $\mathbb{R}^{d}$. Then $\mathbb{S}^{d-1}=\cup \Gamma_{i}$, where $\Gamma_{i}$ is the set of directions such that $\mathcal{L}\left(u, Z_{0}\right)$ is the $i$ th vertex of $Z_{0}$ (the ordering of the vertices can be arbitrary). Note that $\mathcal{H}^{(d-1)}\left(\mathbb{S}^{d-1}\right)=\sum \mathcal{H}^{(d-1)}\left(\Gamma_{i}\right)$. Put

$$
H_{Z_{0}}(\Gamma, W)=\sum_{i} \frac{\mathcal{H}^{(d-1)}\left(\Gamma_{i} \cap \Gamma \cap \sigma\left(Z_{0}, W\right)\right)}{\mathcal{H}^{(d-1)}\left(\Gamma_{i}\right)}
$$

where $\sigma\left(Z_{0}, W\right)$ is the spherical image of $Z_{0}$, see [21, p. 77]. In particular, $H_{Z_{0}}\left(\mathbb{U}, \mathbb{R}^{d}\right)$ is the number of vertices of $Z_{0}$ which is denoted by $\nu$, and $\eta(\mathbb{U}, W)$ is the number of exposed vertices inside $W$ in the Boolean model $Z$ with the typical grain $Z_{0}$. Thus, the ergodic theorem establishes the almost sure convergence of the normalized number of the observed exposed vertices, i.e.,

$$
\eta(\mathbb{U}, W) /|W| \rightarrow \lambda(1-p) \mathbf{E} \nu \quad \text { as } \quad W \uparrow \mathbb{R}^{d} .
$$

The central limit theorem is valid if $\nu$ and $\left|Z_{0}\right|$ have finite second moments. In order to compute the limiting variance consider vertices of $Z_{0}$ to be a (non-stationary) point process $\Psi_{Z_{0}}$ with a.s. finite number of points equal to $\nu$. Its second-order moment measure is denoted by $\alpha^{(2)}(d(x, y))$. Then (7.2) yields

$$
\begin{aligned}
\sigma_{\mathbb{U} U}^{2}=\lambda(1-p)^{2} \int_{\left(\mathbb{R}^{d}\right)^{2}} q(x-y) \alpha^{(2)}(d(x, y)) \\
\quad+\lambda^{2}(1-p)^{2} \int_{\mathbb{R}^{d}}\left(q(x) f(x) f(-x)-(\mathbf{E} \nu)^{2}\right) d x,
\end{aligned}
$$

where

$$
f(x)=\mathbf{E}\left[\sum_{Y_{i} \in \Psi_{Z_{0}}} \mathbf{1}_{Z_{0}^{c}}\left(Y_{i}-x\right)\right] .
$$

ExAmple 9.11. Let $H_{K}(\Gamma, W)$ be equal to the number of the isolated points in compact set $K \subset W$ (clearly, $H_{K}(\Gamma, W)$ does not depend on $\Gamma$ ). Note that $K$ is not supposed to be convex. Then $\eta(\Gamma, W)$ is the number of the exposed isolated points in the Boolean model with the grain $Z_{0}$. Similarly to Example 9.10 , let $\Psi_{Z_{0}}$ be the point process of isolated points of $Z_{0}$ with $\nu$ being the number of points in $\Psi_{Z_{0}}$. Then

$$
\mathbf{E} \eta(\Gamma, W)=\lambda(1-p)|W| \mathbf{E} \nu
$$

If $\mathbf{E} \nu^{2}<\infty$ and $\mathbf{E}\left|Z_{0}\right|^{2}<\infty$, then the central limit theorem is also valid with the covariance given by (9.12). 
EXAmple 9.12. Let $H_{Z_{0}}^{(r)}(\Gamma, W)=\bar{\mu}_{r}\left(Z_{0}, \Gamma \times \mathbb{R}^{d}\right)$, see (8.3). Then the corresponding measure $\eta^{(r)}(\Gamma, W)$ coincides with $\bar{\mu}_{r}\left(Z, \Gamma \times \mathbb{R}^{d}\right)$. As in (8.4),

$$
\eta^{(r)}(\Gamma, W)=\frac{1}{d} \sum_{j=0}^{d-1} r^{d-j}\left(\begin{array}{l}
d \\
j
\end{array}\right) \eta_{j}(\Gamma, W)
$$

where $\eta_{j}(\Gamma, W), j=0, \ldots, d-1$, are measures defined by $(3.7)$ for $H_{K}^{j}(\Gamma, W)=\Theta_{j}(K, \Gamma \times$ $W), K \in \mathcal{C}$. We can use $(9.2)$ to define the corresponding measures $\tilde{\eta}_{j}\left(\Gamma, W_{n}\right)$. Suppose that $\mathbf{E}\left|Z_{0}^{r}\right|^{2}<\infty$ for some $r>0$.

Note that, similarly to the standard Cramér-Wold device, a random vector

$$
\left(\tilde{\eta}_{0}\left(\Gamma, W_{n}\right), \ldots, \tilde{\eta}_{d-1}\left(\Gamma, W_{n}\right)\right)
$$

converges in distribution to $\left(\xi_{0}, \ldots, \xi_{d-1}\right)$ as $n \rightarrow \infty$, if, for each $r>0$,

$$
\sum_{j=0}^{d-1} r^{j} \tilde{\eta}_{j}\left(\Gamma, W_{n}\right)
$$

converges to $\sum_{j=0}^{d-1} r^{j} \xi_{j}$ weakly as $n \rightarrow \infty$. After applying this to the polynomial expansions (9.13) and (8.4), one can prove that the random vector $\left(\tilde{\eta}_{0}\left(\Gamma, W_{n}\right), \ldots, \tilde{\eta}_{d-1}\left(\Gamma, W_{n}\right)\right)$ converges in distribution to a centred Gaussian random vector with the covariances given by

$$
\sigma_{i j}=\lambda \int_{\left(\mathbb{R}^{d}\right)^{2}} q\left(y_{1}-y_{2}\right) \bar{H}_{i j}\left(\Gamma, d y_{1} ; \Gamma, d y_{2}\right)+\lambda^{2} \int_{\mathbb{R}^{d}} \tilde{\Phi}_{i}(x, \Gamma) \tilde{\Phi}_{j}(-x, \Gamma) q(x) d x
$$

where $i, j=0, \ldots, d-1$,

$$
\bar{H}_{i j}\left(\Gamma, W_{1} ; \Gamma, W_{2}\right)=\mathbf{E}\left[\Theta_{i}\left(Z_{0}, \Gamma \times W_{1}\right) \Theta_{j}\left(Z_{0}, \Gamma \times W_{1}\right)\right]
$$

and

$$
\tilde{\Phi}_{i}(x, \Gamma)=\mathbf{E}\left[\Theta_{i}\left(Z_{0}, \Gamma \times\left(Z_{0}+x\right)\right)\right]
$$

\section{Concluding Remarks}

To conclude with, we outline several possible generalizations. We give only the results for the first moments in the Poisson case, although a laborious application of the methods developed above (with evident changes) allows to derive the corresponding limit theorems.

Consider a family of measures $H_{K}^{B}(\cdot), K, B \in \mathcal{C}$, on $\mathfrak{B}(\Sigma)$ which satisfy the conditions

$$
H_{K}^{B}(\Gamma, W)=H_{K}^{B}(\Gamma, W \cap K \cap B) \quad \text { and } \quad H_{K+x}^{B+x}(\Gamma, W+x)=H_{K}^{B}(\Gamma, W), \quad x \in \mathbb{R}^{d} .
$$

In contrast to the standard case, $H_{K}^{B}(\Gamma, W)$ need not be a $\sigma$-finite measure with respect to $B$. For instance, it may be one of the Hausdorff measures or a capacity. The corresponding measure $\eta^{B}(\cdot)$ is defined by

$$
\eta^{B}(\Gamma, W)=\sum_{i: i \geq 1} H_{X_{i}+Z_{i}}^{B}\left(\Gamma, W \backslash \Xi_{i}\right)
$$


Under the basic assumptions,

$$
\mathbf{E} \eta^{B}(\Gamma, W)=\lambda(1-p) \int_{\mathbb{R}^{d}} \mathbf{E} H_{Z_{0}+x}^{B}(\Gamma, W) d x .
$$

ExAmple 10.1. Let $H_{K}^{B}(\Gamma, W)=\Theta_{d-1}((K \cap B), \Gamma \times W)$, i.e., $H_{K}^{B}(\Gamma, W)$ is equal to the $(d-1)$ th curvature measure of the set $K \cap B$. Then for $\Gamma=\mathbb{S}^{d-1}, \eta^{W}(\Gamma, W)$ is equal to the sum of the surface area of $(\partial Z) \cap W$ and the $(d-1)$-dimensional Hausdorff measure of the set of points in $\partial W$ covered by only one grain. Since $H_{K}^{W}(\Gamma, W)=H_{K}^{W}\left(\Gamma, \mathbb{R}^{d}\right)$, (10.1) yields

$$
\mathbf{E} \eta^{W}(\Gamma, W)=\lambda(1-p) \int_{\mathbb{R}^{d}} \mathbf{E} \Theta_{d-1}\left(\left(Z_{0}+x\right) \cap W, \Gamma \times \mathbb{R}^{d}\right) d x .
$$

Now the translative integral formula [21, p. 254] can be applied to get

$$
\begin{aligned}
\mathbf{E} \eta^{W}\left(\mathbb{S}^{d-1}, W\right) & =\lambda(1-p) 2 \mathbf{E}\left[\int_{\mathbb{R}^{d}} V_{d-1}\left(\left(Z_{0}+x\right) \cap W\right) d x\right] \\
& =\lambda(1-p)\left[2 \mathbf{E} V_{d-1}\left(Z_{0}\right)|W|+2 V_{d-1}(W) \mathbf{E}\left|Z_{0}\right|\right] .
\end{aligned}
$$

Remember that $2 V_{d-1}(K), K \in \mathcal{C}$, is equal to the surface area of $K$.

Let us consider the measure $H_{K_{1}, K_{2}}(\cdot)$ which depends on two compact sets $K_{1}$ and $K_{2}$ in such a way that, for all $x \in \mathbb{R}^{d}$,

$$
H_{K_{1}+x, K_{2}+x}(\Gamma, W+x)=H_{K_{1}, K_{2}}(\Gamma, W)=H_{K_{1}, K_{2}}\left(\Gamma, W \cap K_{1} \cap K_{2}\right) .
$$

Then put

$$
\eta(\Gamma, W)=\sum_{i, j \geq 1, i \neq j} H_{Z_{i}+X_{i}, Z_{j}+X_{j}}\left(\Gamma, W \backslash \Xi_{i j}\right)
$$

where

$$
\Xi_{i j}=\bigcup_{k: k \neq i, j}\left(X_{k}+Z_{k}\right)
$$

Then the first moment of $\eta(\cdot)$ is given by

$$
\mathbf{E} \eta(\Gamma, W)=\lambda|W| \int_{\mathbb{R}^{d}} \int_{\mathbb{R}^{d}} G_{0, z}^{!}[f(\cdot)-y] \bar{H}_{z}(\Gamma, d y) \alpha_{\mathrm{red}}^{(2)}(d z),
$$

where

$$
\bar{H}_{z}(\Gamma, W)=\mathbf{E} H_{Z_{1}, Z_{2}+z}(\Gamma, W)
$$

for two independent grains $Z_{1}$ and $Z_{2}$ (we suppose that $\bar{H}_{z}\left(\Gamma, \mathbb{R}^{d}\right)$ is finite for all $z \in \mathbb{R}^{d}$ ). If the point process of germs is Poisson, then

$$
\mathbf{E} \eta(\Gamma, W)=\lambda(1-p)|W| \mathbf{E}\left[\int_{\mathbb{R}^{d}} H_{Z_{1}, Z_{2}+z}\left(\Gamma, \mathbb{R}^{d}\right) d z\right] .
$$


ExAmple 10.2. Let us suppose that $Z$ is the Boolean model with a.s. convex grains. Consider the measure

$$
H_{K_{1}, K_{2}}(\Gamma, W)=\mathbf{1}_{K_{1} \cap K_{2} \cap W \neq \emptyset}, \quad K_{1}, K_{2} \in \mathcal{C}
$$

which satisfies (10.2). Then,

$$
\bar{H}_{z}\left(\Gamma, \mathbb{R}^{d}\right)=\int_{\mathbb{R}^{d}} \mathbf{1}_{Z_{1} \cap\left(Z_{2}+z\right) \neq \emptyset} d z=\left|Z_{1} \oplus \check{Z}_{2}\right|
$$

whence

$$
\mathbf{E} \eta(\Gamma, W)=\lambda(1-p)|W| \mathbf{E}\left|Z_{1} \oplus \check{Z}_{2}\right|
$$

For instance, if $Z_{0}=M$ is a deterministic central symmetric convex set, then (10.3) yields

$$
\mathbf{E} \eta(\Gamma, W)=\lambda(1-p) 2^{d}|W||M|
$$

To give a geometric interpretation of the measure $\eta$ in the planar case remember that two convex planar sets $X_{i}+Z_{i}$ and $X_{j}+Z_{j}$ may be either disjoint or have their boundaries intersected at two points (these sets may touch with probability zero). Then $2 \eta\left(\mathbb{S}^{d-1}, W\right)$ gives the number of such points in $W$ (resulted from $\left(X_{i}+Z_{i}\right) \cap\left(X_{j}+Z_{j}\right)$ ) generated by pairs of grains which are also exposed (not covered by $X_{k}+Z_{k}$ with $k \neq i, j$ ).

Clearly, it is possible to consider also vector-valued measures $H_{K}(\cdot)$. In fact, many results can be generalized also for the germ-grain model $Z$ generated by a not necessarily independently marked point process. Unfortunately, in this case formulae for the variances are getting incomprehensible.

The crucial point of the developed technique is the representation of the measure $\eta$ as the sum of contributions of exposed parts of the grains. Roughly speaking, this means that other grains only determine which part of the given grain contributes to the sum. This excludes possibilities to consider additive extensions of intrinsic volumes of orders $j=0, \ldots, d-2$. Indeed, the additively extended intrinsic volumes, $V_{j}(F), F \in \mathcal{R}$, are defined by an analogue of (8.1), so that their values depend on the angles of intersections of the convex sets which give $F$ as the union-set. For instance, it is not possible to obtain the Euler-Poincaré characteristic as a special case of the measure $\eta$ given by (3.7). 


\section{REFERENCES}

[1] A.J. Baddeley. Absolute curvatures in integral geometry. Math. Proc. Cambridge Philos. Soc., 88:45-58, 1980.

[2] A.J. Baddeley. A limit theorem for statistics of spatial data. Adv. in Appl. Probab., 12:447-461, 1980.

[3] R.N. Bhattacharia and R. Ranga Rao. Normal Approximation and Asymptotic Expansions. Wiley, New York, 1976.

[4] D.J. Daley and D. Vere-Jones. An Introduction to the Theory of Point Processes. Springer, New York, 1988.

[5] F. Götze, L. Heinrich, and C. Hipp. m-dependent random fields with analytical cumulant generating function. Scand. J. Statist., 22:183-195, 1995.

[6] H. Hadwiger. Vorlesungen über Inhalt, Oberfläche und Isoperimetrie. Springer, Berlin, 1957.

[7] P. Hall. Introduction to the Theory of Coverage Processes. Wiley, New York, 1988.

[8] K.-H. Hanish. On classes of random sets and point processes. Serdica, 7:160-166, 1981.

[9] L. Heinrich. Asymptotic behaviour of an empirical nearest-neighbour distance function for stationary Poisson cluster process. Math. Nachr., 136:131-148, 1988.

[10] L. Heinrich. Asymptotic Gaussianity of some estimators for reduced factorial moment measures and product densities of stationary Poisson cluster processes. statistics, 19:87-106, 1988 .

[11] L. Heinrich. On existence and mixing properties of germ-grain models. statistics, 23:271-286, 1992.

[12] L. Heinrich. Asymptotic properties of minimum contrast estimators for parameters of Boolean models. Metrika, 31:349-360, 1993.

[13] L. Heinrich. Normal approximation for some mean-value estimates of absolutely regular tressellations. Math. Methods of Statist., 3:1-24, 1994.

[14] O. Kallenberg. Random Measures. Akademie-Verlag/Academic Press, Berlin/New York, third edition, 1983.

[15] S. Mase. Asymptotic properties of stereological estimators of volume fraction for stationary random sets. J. Appl. Probab., 19:111-126, 1982.

[16] G. Matheron. Random Sets and Integral Geometry. Wiley, New York, 1975.

[17] I.S. Molchanov. Statistics of the Boolean model: From the estimation of means to the estimation of distributions. Adv. in Appl. Probab., 27:63-86, 1995. 
[18] I.S. Molchanov and D. Stoyan. Asymptotic properties of estimators for parameters of the Boolean model. Adv. in Appl. Probab., 26:301-323, 1994.

[19] X.X. Nguyen and H. Zessin. Ergodic theorems for spatial processes. Z. Wahrsch. verw. Gebiete, 48:133-158, 1979.

[20] R. Schneider. Parallelmengen mit Vielfachheit und Steiner-Formeln. Geom. Dedicata, 2:111-127, 1980.

[21] R. Schneider. Convex Bodies. The Brunn-Minkowski Theory. Cambridge Univ. Press, Cambridge, 1993.

[22] J. Serra. Image Analysis and Mathematical Morphology. Academic-Press, London, 1982.

[23] D. Stoyan, W.S. Kendall, and J. Mecke. Stochastic Geometry and Its Applications. Wiley, Chichester, 1987.

[24] V.A. Volkonskiŭ and Yu.A. Rozanov. Some limit theorems for random functions. Theory Probab. Appl., 4:178-197, 1959.

[25] W. Weil. Stereology: A survey for geometers. In P.M. Gruber and J.M. Wills, editors, Convexity and Its Applications, pages 360-412. Birkhäuser, Basel etc., 1983.

[26] W. Weil. Expectation formulas and isoperimetric properties for non-isotropic Boolean models. J. Microscopy, 151:235-245, 1988.

[27] W. Weil and J.A. Wieacker. Densities for stationary random sets and point processes. Adv. in Appl. Probab., 16:324-346, 1984.

[28] M. Zähle. Random processes of Hausdorff rectifiable closed sets. Math. Nachr., 108:49-72, 1982.

[29] M. Zähle. Curvature measures and random sets, I. Math. Nachr., 119:327-339, 1984.

[30] M. Zähle. Curvature measures and random sets, II. Probab. Theor. Relat. Fields, 71:37-58, 1986.

[31] M. Zähle. Absolute curvature measures. Math. Nachr., 140:83-90, 1989. 